\title{
A Variant of the Mountain Pass Theorem and Variational Gluing
}

\author{
Piero Montecchiari and Paul H. Rabinowitz
}

\begin{abstract}
This paper surveys some recent work on a variant of the Mountain Pass Theorem that is applicable to some classes of differential equations involving unbounded spatial or temporal domains. In particular its application to a system of semilinear elliptic PDEs on $\mathbb{R}^{n}$ and to a family of Hamiltonian systems involving double well potentials will also be discussed.
\end{abstract}

Mathematics Subject Classification (2010). Primary: 35J50, 35J47; Secondary: 35J57, 34C37.

Keywords. Variational methods, mountain pass theorem, variational gluing, nondegeneracy condition, heteroclinic solutions, homoclinic solutions, double well potential, multitransition solutions.

\section{Introduction}

The Mountain Pass Theorem is a useful tool for obtaining the existence of solutions of differential equations that arise as critical points of a functional, $I$, defined on a Banach space, $E$, or a subset thereof. The differential equations in their weak form correspond to $I^{\prime}(u)=0, I^{\prime}$ denoting the Frechet derivative of $I$. The functional is required to possess an appropriate geometric structure. The simplest example of that structure occurs when $I$ has a local minimum that is not a global minimum. The other requirements of the Theorem are some smoothness for $I$, e.g. $I \in C^{1}(E, \mathbb{R})$, and some compactness such as is embodied in the Palais-Smale condition, $(P S)$ : any sequence for which $I$ is bounded and $I^{\prime} \rightarrow 0$ possesses a convergent subsequence. The Mountain Pass Theorem also provides a minimax characterization of the associated critical value, $c$ :

$$
c=\inf _{g \in \Gamma} \max _{\theta \in[0,1]} I(g(\theta))
$$

where

$$
\Gamma=\{g \in C([0,1], E) \mid g(0)=0 \text { and } g(1)=e\}
$$

and the local minimum of $I$ occurs at 0 with $\mathrm{I}(0)=0$ and $e \neq 0$ is such that $I(e) \leq 0$. 
In most applications, $E$ is a class of functions having a bounded temporal or spatial domain. However if the domain is unbounded, even if the smoothness and geometric conditions are satisfied, the $(P S)$ condition may fail. Two examples in which this happens will be considered in Section 2. The first example involves a family of semilinear elliptic equations on $\mathbb{R}^{n}$ of the form:

$$
-\Delta u+u=F_{u}(x, u), \quad x \in \mathbb{R}^{n} .
$$

A model case here is $F=a(x)|u|^{p-1} u$ where $1<p<\frac{n+2}{n-2}$ with $n>2$ or $1<p<+\infty$ when $n=1,2$, and $a(x)$ is periodic in the each of the components of $x$. The second example is a family of second order Hamiltonian systems with a double well potential on $\mathbb{R}^{m}$ :

$$
-\ddot{q}+V_{q}(t, q)=0, t \in \mathbb{R}, q \in \mathbb{R}^{m} .
$$

where $V$ is 1-periodic in $t$. Geometrically in Example 1, the associated functional, $I$, has 0 as a local but not global minimum while in Example 2, $I$ has a pair of global minima at the minima of the potential. Moreover due to the periodicity of $a$ and $V$, the associated functional, $I$ is invariant under a translational symmetry. This additional information together with the aid of concentration compactness arguments allows one to analyze how $(P S)$ breaks down. In the process, one finds that $I^{\prime}=0$ must possess at least one additional solution (modulo the translational symmetry). We will refer to these new solutions as the basic solutions of $I^{\prime}=0$. There is an associated family of critical values. Understanding the breakdown of $(P S)$ also led to the development of variational gluing arguments that establish the existence of so-called multibump and multitransition solutions of $I^{\prime}=0$ in Examples 1 and 2 respectively, provided that the set of basic solutions satisfies a mild non-degeneracy condition.

A third example in which the above phenomena and results occur is the system

$$
-\Delta u+G_{u}(x, u)=0
$$

where $G$ is periodic in the components of $x \in \mathbb{R}^{n}$ and $u \in \mathbb{R}^{m}$. It will be discussed in Section 6.

Our goal in this paper is to survey some recent work on a variant of the Mountain Pass Theorem that can be used to obtain the existence of basic mountain pass solutions in several settings such as the first two examples above and to indicate how the variational methods can be further employed to "glue" these solutions to construct multibump and multitransition solutions of $I^{\prime}=0$ such as mentioned above. For such constructions as well as to obtain the basic mountain pass solutions themselves, some sort of nondegeneracy condition is generally required and such conditions will also be introduced and discussed.

The new mountain pass result will be presented in Section 3. As will be shown via an example, a consequence of this result is that, unlike the original version, the minimax value, $c$, in (1.1) may not be a critical value of $I$, but instead a limit of critical values. In fact this is indicative of the role that nondegeneracy conditions play in variational gluing and what one finds in applications. Although we will not always express it in that fashion, a natural alternative occurs: Either there is a 
continuum of basic solutions (this being the degenerate case) or a variational gluing construction can be made resulting in infinitely many multibump or multitransition solutions of $I^{\prime}=0$. In Section 4, we indicate how the new mountain pass result can be applied to (HS) and in Section 5, to (PDE).

There is a sizable literature on the topics just mentioned. The Mountain Pass Theorem as stated above is due to Ambrosetti and Rabinowitz [1]. Some other papers that give more information about the topological nature of a mountain pass critical point are due to Pucci and Serrin [2]-[4], Hofer [5] and Caldiroli and Montecchiari [6]. The variant that is described in Section 3 appears in [7]. The relationship between various nondegeneracy conditions was treated in [8]. See also Cieliebak and Séré [9].

The system (PDE) and variants thereof have been extensively studied. In a bounded domain, the related single equation was treated in [1]. For some earlier related work, see Coffman [10]-[11] and Hemple [12]-[13] as well as the references in [1]. The single equation on an unbounded domain was studied in Coti Zelati and Rabinowitz [14], in Alama and Li [21] and in Montecchiari [15]. The more general related system of equations, (PDE) was treated in [16] and [17]. Variational gluing was a key goal of [14] and was motivated by work of Séré on first order Hamiltonian systems [18]-[19] and the work of Coti Zelati and Rabinowitz on second order Hamiltonian systems [20]. See also [22].

As to (HS), some early work using variational methods to find connecting orbit of Hamiltonian systems can be found in Mather [23]-[24], Bolotin [25], and Kozlov [26]. Specializing to double well potentials, see e.g. Sternberg [27]. The approach taken here is based on Byeon, Montecchiari, and Rabinowitz [28], and on [7]. See also Alikakos and Fusco [29] and Alessio, Montecchiari, and Zuniga [30].

For the scalar setting of (Per), i.e. the case of $m=1$, a more general version of this equation was introduced by Moser [31] and furthered by Bangert [32]. An extensive collection of results for the scalar version of (Per) can be found in the monograph [33] of Rabinowitz and Stredulinsky. These results do not include solutions of mountain pass type. Such results can be found in Bolotin and Rabinowitz [34]-[35]. The system version of (Per) has been studied in [36].

\section{Two examples}

In this section, two examples will be given for which there is mountain pass structure but $(P S)$ fails to hold. Nevertheless there still exist solutions of mountain pass type as well as more complicated multibump or multitransition solutions. The first example is (PDE) from the Introduction where solutions are sought in $E=W^{1,2}\left(\mathbb{R}^{n}, \mathbb{R}\right)$. The corresponding functional is

$$
I(u)=\int_{\mathbb{R}^{n}}\left(\frac{1}{2}\left(|\nabla u|^{2}+u^{2}\right)-F(x, u)\right) d x .
$$

The function $F$ satisfies

$\left(F_{1}\right) F \in C^{2}\left(\mathbb{R} \times \mathbb{R}^{n}, \mathbb{R}\right)$ and is 1 -periodic in the components of $x=\left(x_{1}, \ldots, x_{n}\right)$. 
$\left(F_{2}\right)$ There is a constant $a_{1}>0$ such that

$$
\left|F_{u u}(x, z)\right| \leq a_{1}\left(1+|z|^{p-1}\right) \text { if } 1<p<\frac{n+2}{n-2} \text { for } n>2 \text { and } p>1 \text { if } n=1,2 .
$$

$\left(F_{3}\right) F_{u u}(x, 0)=0$ for $x \in \mathbb{R}^{n}$.

$\left(F_{4}\right)$ There is a constant, $\mu \in\left(0, \frac{1}{2}\right)$ such that

$$
0<F(x, z)<\mu z \cdot F_{u}(x, z) \text { for } x \in \mathbb{R}^{n} \text { and } z \neq 0 \text {. }
$$

Problems for (PDE) under $\left(F_{1}\right)-\left(F_{4}\right)$ arise on seeking standing wave solutions of nonlinear Schrödinger equations. As was shown in [14], assuming conditions $\left(F_{1}\right)-$ $\left(F_{4}\right)$, then $I \in C^{2}(E, \mathbb{R})$ and critical points of $I$ in $E$ are classical solutions of (PDE). In fact, under a milder set of conditions than $\left(F_{1}\right)-\left(F_{4}\right)$, if $\mathbb{R}^{n}$ is replaced by a bounded subdomain $\Omega$, and $u$ is required to vanish on $\partial \Omega$, then as one of the original applications of the Mountain Pass Theorem, it was proved in [1] that there exists a solution to this modified version of (PDE). Moreover $c$ as defined in (1.1) is a critical value of $I$.

Returning to the unbounded case, it is easy to see that the functional, $I$ does not satisfy $(P S)$. Indeed if $(P S)$ were to hold, the Mountain Pass Theorem would be valid for this setting and $c$ as defined in (1.1) would be a critical value of $I$. Let $u$ be a corresponding critical point. Due to $\left(F_{1}\right), I(u(\cdot+k))=I(u)=c$ for all $k \in \mathbb{Z}$. Set $u_{k}=u(\cdot+k)$. Then $I\left(u_{k}\right)$ is bounded and $I^{\prime}\left(u_{k}\right)=0$ so $\left(u_{k}\right)$ is a Palais-Smale sequence. Hence by the $(P S)$ condition, it possesses a convergent subsequence. But $u_{k}$ converges weakly and not strongly to 0 , a contradiction. Nevertheless it is still the case that $I$ possesses nonzero critical points. One natural approach to proving this is to approximate $\mathbb{R}^{n}$ by a sequence of increasing periodic domains, $\Omega_{k}$, to which the result of [1] applies, use the fact that if $U_{k}$ is a solution of (PDE), so is $U_{k}(\cdot+j)$ for any $j \in \mathbb{Z}^{n}$ to put the maximum of $\left|U_{k}\right|$ in $[0,1]^{n}$, get bounds for the corresponding critical points, $U_{k}$, and show that as $k \rightarrow \infty$, a subsequence of $U_{k}$ possesses a limit, $U \not \equiv 0$ with $U$ satisfying (PDE). Such a strategy was successfully used in [37] for $I$.

A disadvantage of the approach just described it is that it gives no variational characterization of the solution, $U$, or $I(U)$. E.g. in [37], one can only conclude that $I(U) \leq c$. More information is needed to establish the existence of multibump solutions of (PDE) which was the goal of [14]. To explain what we mean by a multibump solution of (PDE), note that although $I$ under $\left(F_{1}\right)-\left(F_{4}\right)$ does not satisfy $(P S)$, it still possesses a remnant of that condition as the next result shows. First we recall that in [14] or [8], it was shown that there is a $c_{0}>0$ such that if $U \not \equiv 0$ is a solution of (PDE), $I(U) \geq c_{0}$. For $s \in \mathbb{R}$, set

$$
I^{s}=\{u \in E \mid I(u) \leq s\}, \mathcal{D}=\left\{u \in E \backslash\{0\} \mid I^{\prime}(u)=0\right\} \text {, and } \mathcal{D}^{s}=\mathcal{D} \cap I^{s} .
$$

Proposition 2.2. [14] Suppose that $F$ satisfies $\left(F_{1}\right)-\left(F_{4}\right)$. Let $\left(u_{k}\right) \subset E$ be such that $I\left(u_{k}\right) \rightarrow b \geq c_{0}$ and $I^{\prime}\left(u_{k}\right) \rightarrow 0$ as $k \rightarrow \infty$. Then there exists $a \beta \in \mathbb{Z} \cap\left[0, \frac{b}{c_{0}}\right]$, $U_{0} \in(\{0\} \cup \mathcal{D}) \cap I^{b}, U_{1}, \ldots, U_{\beta} \in \mathcal{D} \cap I^{b}$, a subsequence of $\left(u_{k}\right)$, again denoted by $\left(u_{k}\right)$, and sequences $\left(\xi_{k}^{1}\right), \ldots,\left(\xi_{k}^{\beta}\right) \in \mathbb{Z}^{n}$ such that, as $k \rightarrow \infty,\left|\xi_{k}^{j}\right| \rightarrow \infty$ for any 


$$
\begin{gathered}
j \in\{1, \ldots, \beta\},\left|\xi_{k}^{i}-\xi_{k}^{j}\right| \rightarrow+\infty \quad i \neq j, b=\sum_{j=0}^{\beta} I\left(U_{j}\right), \text { and } \\
\left\|u_{k}-U_{0}-\sum_{j=1}^{\beta} U_{j}\left(\cdot-\xi_{k}^{j}\right)\right\| \rightarrow 0 .
\end{gathered}
$$

Stated more informally, this Proposition says along a subsequence, a (PS) sequence converges to a formal chain of widely spaced translates of solutions of (PDE), each of which corresponds to a critical point of $I$ that is less than or equal to $b$. A result like Proposition 2.2 in the setting of a first order Hamiltonian system can be found in Lemma 3.3 of the work of Coti Zelati, Ekeland, and Séré [38] and was based on the notion of concentration compactness introduced by P.L. Lions in [39]-[40]. See also the further work of Séré on first order Hamiltonian systems: [18]-[19].

It is worth noting at this point that solutions of $(\mathrm{PDE})$ in $W^{1,2}\left(\mathbb{R}^{n}, \mathbb{R}\right)$ decay exponentially to 0 as $|x| \rightarrow \infty$. Consequently for large $k$, the sum term in (2.3) can be viewed geometrically as consisting of $\beta$ bumps. Thus we say a multibump solution of (PDE) is an actual solution that is near such a finite (or possibly infinite) sum of solutions. In the terminology of dynamical systems, a multibump solution shadows such a sum of solutions.

As was mentioned above, the existence of multibump solutions of (PDE) was the main goal of [14]. The first step in the argument there is to show the minimax value, $c$, of (1.1) is actually a critical value of $I$. To accomplish this, another assumption was required in [14], namely

$$
\text { There is an } \alpha>0 \text { such that } \mathcal{D}^{c+\alpha} / \mathbb{Z}^{n} \text { is finite. }
$$

This condition, which is the analogue of a similar requirement in [18], together with $\left(F_{1}\right)-\left(F_{4}\right)$ and a variant of the usual sort of deformation argument associated with the Mountain Pass Theorem leads to the following result:

Theorem 2.4. [14] If $F$ satisfies $\left(F_{1}\right)-\left(F_{4}\right)$ and $\left(N D_{1}\right)$ holds, $c$ is a critical value of $I$.

The assumption $\left(N D_{1}\right)$ leads to topological information about the behavior of the functional $I$ at values near $c$. This topological information plays an important role in the construction of multibump solutions. Proposition 3.2 below describes a generalization of these topological properties and states their validity under weak nondegeneracy assumptions.

As our second example, consider the second order Hamiltonian system:

$$
-\ddot{q}+V_{q}(t, q)=0, \quad q \in \mathbb{R}^{m}
$$

where $V$ is a double well potential satisfying

$\left(V_{1}\right) V \in C^{1}\left(\mathbb{R} \times \mathbb{R}^{m}, \mathbb{R}\right)$ and is 1 -periodic in $t \in \mathbb{R}$.

$\left(V_{2}\right)$ There are points $a^{-}, a^{+} \in \mathbb{R}^{m}$ where $V(t, q)>V\left(t, a^{ \pm}\right)=0$ for any $q \in$ $\mathbb{R}^{m} \backslash\left\{a^{-}, a^{+}\right\}$.

$\left(V_{3}\right)$ There is a constant, $V_{0}>0$, such that $\liminf _{|q| \rightarrow+\infty} V(t, q) \geq V_{0}$. 
The Lagrangian for (HS) is $L(q)=\frac{1}{2}|\dot{q}|^{2}+V(t, q)$. The associated functional is

$$
J(q)=\int_{\mathbb{R}} L(q) d t .
$$

Note that by $\left(V_{1}\right), J$ is invariant under the family of phase shifts, $q(t) \rightarrow q(t+k)$ for all $k \in \mathbb{Z}$. Minimizing $J$ on $W_{l o c}^{1,2}\left(\mathbb{R}, \mathbb{R}^{m}\right)$ produces the equilibrium solutions, $a^{-}$and $a^{+}$of (HS). They correspond to the solution, $u=0$, of (PDE) that is a local minimum of $I$ in (2.1). However for (HS), there is an infinitude of additional solutions that can be obtained via minimization arguments.

To set up a framework to obtain these new solutions, for $i \in \mathbb{Z}$, let $T_{i}=[i, i+1]$. As the underlying space here, we take

$$
\mathcal{E} \equiv\left\{\left.q \in W_{l o c}^{1,2}\left(\mathbb{R}, \mathbb{R}^{m}\right)\left|\int_{\mathbb{R}}\right| \dot{q}\right|^{2} d t+\int_{T_{0}}|q|^{2} d t<\infty\right\} .
$$

Then $\mathcal{E}$ is a Hilbert space under the inner product associated with the norm

$$
\|q\|^{2}=\int_{\mathbb{R}}|\dot{q}|^{2} d t+\int_{T_{0}}|q|^{2} d t .
$$

Since (HS) has $a^{-}$and $a^{+}$as equilibrium solutions, it is natural to seek solutions of the equation that are heteroclinic from $a^{-}$to $a^{+}$or from $a^{+}$to $a^{-}$. As was mentioned in the Introduction, this problem has been extensively studied. We briefly recall some results from [28].

Consider $J$ on $\mathcal{E}$ and define

$$
\Gamma\left(a^{-}, a^{+}\right)=\left\{q \in \mathcal{E} \mid q( \pm \infty)=a^{ \pm}\right\}
$$

By $q( \pm \infty)=a^{ \pm}$, we mean $\lim _{t \rightarrow \pm \infty} q(t)=a^{ \pm}$. This condition is equivalent to the requirement $\lim _{i \rightarrow \pm \infty}\left\|q-a^{ \pm}\right\|_{L^{2}\left(T_{i}, \mathbb{R}^{m}\right)}=0$ in [28]. Define

$$
c\left(a^{-}, a^{+}\right)=\inf _{q \in \Gamma\left(a^{-}, a^{+}\right)} J(q)
$$

and set

$$
\mathcal{M}\left(a^{-}, a^{+}\right)=\left\{q \in \Gamma\left(a^{-}, a^{+}\right) \mid J(q)=c\left(a^{-}, a^{+}\right)\right\} .
$$

Then we have:

Theorem 2.7. Suppose that $\left(V_{1}\right)-\left(V_{3}\right)$ are satisfied. Then $\mathcal{M}\left(a^{-}, a^{+}\right) \neq \emptyset$ and any $Q \in \mathcal{M}\left(a^{-}, a^{+}\right)$is a $C^{2}$ solution of (HS) heteroclinic from $a^{-}$to $a^{+}$.

Remark 2.8. (i) Reversing the roles of $a^{-}$and $a^{+}$in $\Gamma\left(a^{-}, a^{+}\right), c\left(a^{-}, a^{+}\right)$and $\mathcal{M}\left(a^{-}, a^{+}\right)$yields solutions of (HS) heteroclinic from $a^{+}$to $a^{-}$. (ii) The functional, $J$, is not Fréchét differentiable on $\mathcal{E}$. Therefore the usual argument of working with $J^{\prime}(Q)=0$ to obtain regularity cannot be employed directly and a bit more care is needed to show the members of $\mathcal{M}\left(a^{-}, a^{+}\right) \cup \mathcal{M}\left(a^{+}, a^{-}\right)$are $C^{2}$ functions. (iii) Theorem 2.7 appears as Theorem 2.34 of [28]. The paper [28] studied the existence of solutions of a nonlinear Neumann problem of a system of elliptic partial differential equations in an infinite cylinder in $\mathbb{R}^{n}$, the axis of the cylinder being in the $x_{1}$ direction. When $n=1$, the problem reduces to (HS). 
At this point, there are two possible directions in which to proceed. Either we can continue with the investigation of solutions of (HS) that can be obtained by minimization methods, namely multitransition solutions, or we can begin a study of mountain pass solutions. Both of these directions require making a nondegeneracy assumption for $\mathcal{M}\left(a^{-}, a^{+}\right) \cup \mathcal{M}\left(a^{+}, a^{-}\right)$. We will follow the historical route and first discuss multitransition solutions that are local minima of $J$. In fact, in so doing, later we will be led to further mountain pass solutions of (HS).

Thus let $k \in \mathbb{N}$ with $k \geq 2$. For $k$ odd, a $k$-transition solution of (HS) is a heteroclinic emanating from say $a^{-}$and oscillating $k$ times between $a^{-}$and $a^{+}$ before terminating at $a^{+}$. For $k$ even, in the same fashion, a $k$-transition solution is homoclinic to $a^{-}$(or $a^{+}$). To introduce the nondegeneracy condition needed here, set

$$
\mathcal{S}\left(a^{-}, a^{+}\right)=\left\{\left.Q\right|_{T_{0}} \mid Q \in \mathcal{M}\left(a^{-}, a^{+}\right)\right\} .
$$

The diagram below indicates the elements in $\mathcal{S}$ generated by a single heteroclinic $q$ and its integer phase shifts (see Figure 1).

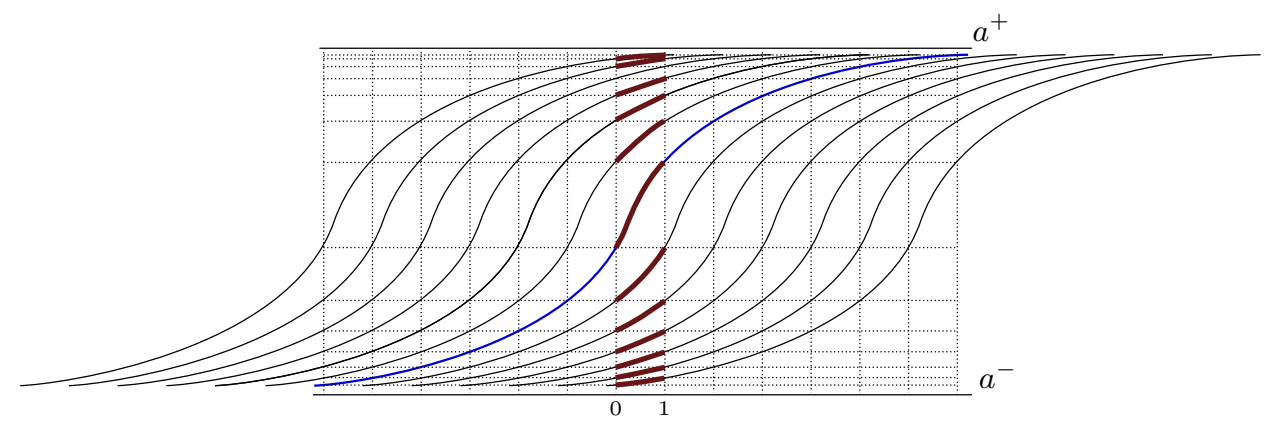

FIGURE 1. A heteroclinic q, its integer phase shifts and their contributions to $\mathcal{S}$

The subset $\mathcal{S}\left(a^{-}, a^{+}\right)$of $W^{1,2}\left(T_{0}, \mathbb{R}^{m}\right)$ may be thought of as the family of unit time snapshots of all possible members of $\mathcal{M}\left(a^{-}, a^{+}\right)$. It possesses the following properties:

- $\overline{\mathcal{S}}\left(a^{-}, a^{+}\right)=\mathcal{S}\left(a^{-}, a^{+}\right) \cup\left\{a^{-}\right\} \cup\left\{a^{+}\right\}$,

- $\overline{\mathcal{S}}\left(a^{-}, a^{+}\right)$is compact in $W^{1,2}\left(T_{0}, \mathbb{R}^{m}\right)$.

See [28] for the details. Let $\mathcal{C}_{a^{-}}\left(a^{-}, a^{+}\right)$denote the component of $\overline{\mathcal{S}}\left(a^{-}, a^{+}\right)$containing $a^{-}$and let $\mathcal{C}_{a^{+}}\left(a^{-}, a^{+}\right)$be the component of $\overline{\mathcal{S}}\left(a^{-}, a^{+}\right)$containing $a^{+}$. Then from e.g. [28], we have the following "All or Nothing" alternative:

\section{Proposition 2.9. Either}

(i) $\mathcal{C}_{a^{-}}\left(a^{-}, a^{+}\right)=\mathcal{C}_{a^{+}}\left(a^{-}, a^{+}\right)$, or

(ii) $\mathcal{C}_{a^{-}}\left(a^{-}, a^{+}\right)=\left\{a^{-}\right\}$and $\mathcal{C}_{a^{+}}\left(a^{-}, a^{+}\right)=\left\{a^{+}\right\}$.

Proof. Since the proof is so simple, we include it here. Suppose (i) and (ii) fail to hold. Then $\mathcal{C}_{a^{-}}\left(a^{-}, a^{+}\right)$does not meet $\mathcal{C}_{a^{+}}\left(a^{-}, a^{+}\right)$and there is a $z \in \mathcal{C}_{a^{-}}\left(a^{-}, a^{+}\right) \backslash\left\{a^{-}\right\}$. Let $k \in \mathbb{Z}$. The map $g^{k}(\zeta)=\zeta(t+k)$ is a continuous map of the compact and 
connected set $\mathcal{C}_{a^{-}}\left(a^{-}, a^{+}\right)$into itself with $g^{k}\left(a^{-}\right)=a^{-}$. But as $k \rightarrow \infty, g^{k}(z) \rightarrow a^{+}$ so $a^{+} \in \mathcal{C}_{a^{-}}\left(a^{-}, a^{+}\right)$, a contradiction.

As an immediate consequence of Proposition 2.9, the next result shows item (ii) implies a useful splitting of $\overline{\mathcal{S}}\left(a^{-}, a^{+}\right)$.

Proposition 2.10. If (ii) of Proposition holds, there exist nonempty disjoint compact sets, $K_{a^{-}}\left(a^{-}, a^{+}\right), K_{a^{+}}\left(a^{-}, a^{+}\right) \subset \overline{\mathcal{S}}\left(a^{-}, a^{+}\right)$such that

(i) $a^{-} \in K_{a^{-}}\left(a^{-}, a^{+}\right), a^{+} \in K_{a^{+}}\left(a^{-}, a^{+}\right)$,

(ii) $\overline{\mathcal{S}}\left(a^{-}, a^{+}\right)=K_{a^{-}}\left(a^{-}, a^{+}\right) \cup K_{a^{+}}\left(a^{-}, a^{+}\right)$,

(iii) $\operatorname{dist}\left(K_{a^{-}}\left(a^{-}, a^{+}\right), K_{a^{+}}\left(a^{-}, a^{+}\right)\right) \equiv 4 r\left(a^{-}, a^{+}\right) \equiv r_{-}>0$.

The splitting, $K_{a^{-}}\left(a^{-}, a^{+}\right), K_{a^{+}}\left(a^{-}, a^{+}\right)$, of $\overline{\mathcal{S}}\left(a^{-}, a^{+}\right)$is not unique. Indeed subjecting each of the functions, $q$, that make up these sets to the same integer phase shift produces a new such splitting. Consider the choice of this splitting to be fixed for what follows. Reversing the roles of $a^{-}$and $a^{+}$yields sets $\mathcal{C}_{a^{+}}\left(a^{+}, a^{-}\right)$, $\mathcal{C}_{a^{-}}\left(a^{+}, a^{-}\right), K_{a^{+}}\left(a^{+}, a^{-}\right), K_{a^{-}}\left(a^{+}, a^{-}\right)$, namely the analogue for heteroclinics from $a^{+}$to $a^{-}$of what we have obtained for heteroclinics from $a^{-}$to $a^{+}$.

The nondegeneracy conditions that we impose are those of alternative (ii) of Proposition 2.9 and its counterpart with $a^{-}$and $a^{+}$reversed:

$$
\begin{gathered}
\mathcal{C}_{a^{-}}\left(a^{-}, a^{+}\right)=\left\{a^{-}\right\}, \mathcal{C}_{a^{+}}\left(a^{-}, a^{+}\right)=\left\{a^{+}\right\}, \mathcal{C}_{a^{+}}\left(a^{+}, a^{-}\right)=\left\{a^{+}\right\} \\
\text {and } \mathcal{C}_{a^{-}}\left(a^{+}, a^{-}\right)=\left\{a^{-}\right\}
\end{gathered}
$$

Note that $\left(N D_{2}\right)$ fails if (HS) is autonomous since then $Q \in \mathcal{M}\left(a^{-}, a^{+}\right)$implies $Q(\cdot+p) \in \mathcal{M}\left(a^{-}, a^{+}\right)$for all $p \in \mathbb{R}$.

The condition $\left(N D_{2}\right)$ and in particular, the splitting properties given in Proposition 2.10, play an important role in formulating a minimization problem to obtain $k$-transition solutions of (HS). Only the case of $k=2$ will be described. The general case is treated similarly. For notational simplicity, let $K_{1}=K_{a^{-}}\left(a^{-}, a^{+}\right), K_{2}=$ $K_{a^{+}}\left(a^{-}, a^{+}\right), K_{3}=K_{a^{+}}\left(a^{+}, a^{-}\right), K_{4}=K_{a^{-}}\left(a^{+}, a^{-}\right)$. Due to $\left(N D_{2}\right)$, by Proposition 2.10, $K_{1}$ and $K_{2}$ are compact, disjoint, and

$$
4 r_{-} \equiv\left\|K_{1}-K_{2}\right\|_{W^{1,2}\left(T_{0}, \mathbb{R}^{m}\right)}>0
$$

and similarly

$$
4 r_{+} \equiv\left\|K_{3}-K_{4}\right\|_{W^{1,2}\left(T_{0}, \mathbb{R}^{m}\right)}>0 \text {. }
$$

Set $\bar{r}=\min \left\{r_{-}, r_{+}\right\}$, so $\bar{r}>0$. Let $\mathbf{m}=\left(m_{1}, \ldots, m_{4}\right) \in \mathbb{Z}^{4}$ and $l \in \mathbb{N}$ satisfy

$$
m_{1}+2 l<m_{2}-2 l<m_{2}+2 l<m_{3}-2 l<m_{3}+2 l<m_{4}-2 l .
$$

For $S \subset W^{1,2}\left(T_{0}, \mathbb{R}^{m}\right)$, and $r>0$, let

$$
N_{r}(S)=\left\{q \in W^{1,2}\left(T_{0}, \mathbb{R}^{m}\right) \mid\|q-S\|_{W^{1,2}\left(T_{0}, \mathbb{R}^{m}\right)} \leq r\right\} .
$$


The class of admissible functions associated with $\mathbf{m}$ and $l$ is $\mathcal{A}_{2}(\mathbf{m}, \mathbf{l})$ where $q \in$ $\mathcal{A}_{2}(\mathbf{m}, l)$ if $0<r \leq \bar{r}$ and

$$
\left.q(\cdot+j)\right|_{T_{0}} \in \begin{cases}N_{r}\left(K_{1}\right), & j<m_{1}+l, \\ N_{r}\left(K_{2}\right), & m_{2}-l \leq j<m_{2}+l, \\ N_{r}\left(K_{3}\right), & m_{3}-l \leq j<m_{3}+l, \\ N_{r}\left(K_{4}\right), & m_{4}-l \leq j .\end{cases}
$$

Thus the members of $\mathcal{A}_{2}(\mathbf{m}, \mathbf{l})$ are constrained to shadow the basic heteroclinic solutions of (HS) in the fashion required by (2.14) (see figure 2).

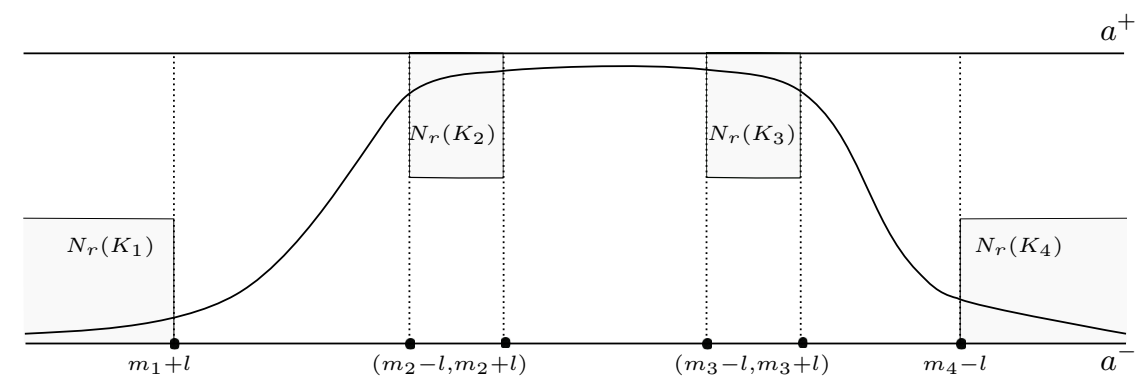

Figure 2. The constrained elements of $\mathcal{A}_{2}(\mathbf{m}, \mathbf{l})$

Define

$$
c(\mathbf{m}, l)=\inf _{q \in \mathcal{A}_{2}(\mathbf{m}, l)} J(q) .
$$

Now we have:

Theorem 2.16. If $\left(V_{1}\right)-\left(V_{3}\right)$ are satisfied and $\left(N D_{2}\right)$ holds, there is an $m_{0} \in \mathbb{N}$ such that if $l \geq m_{0}$ and

$$
m_{i+1}-m_{i}-6 l \geq m_{0} \text { for } i=1,2,3,
$$

then $J$ has a minimizer in $\mathcal{A}_{2}(\mathbf{m}, \mathbf{l})$. Moreover each such minimizer is a solution of (HS) that is homoclinic to $a^{-}$.

Remark 2.18. (i) The flexibility in choosing $\mathbf{m}$ in Theorem 2.16 shows that (HS) possesses infinitely many distinct 2 -transition solutions. (ii) The Theorem is a special case of Theorem 5.16 of [28]. See also Theorem 3.4 in [41]. Due to the setting of that paper, (see our Remark 2.8), an additional growth assumption called $\left(V_{4}\right)$ was required there. It is not needed in our simpler setting.

The detailed proof of Theorem 2.16 can be found in [28]. See also [41] for the proof of a related result. Here we only want to indicate the crucial role that $\left(N D_{2}\right)$ and the splitting properties of $\mathcal{S}\left(a^{-}, a^{+}\right)$and $\mathcal{S}\left(a^{+}, a^{-}\right)$play in the argument. It is straightforward to show for any $m_{0}$ that $J$ has a minimizer, $Q \in \mathcal{A}_{2}$. If $m_{0}$ is large enough, $Q$ satisfies the constraints defining $\mathcal{A}_{2}$ with strict inequalities and therefore by a standard regularity argument, $Q$ is a solution of (HS). That strict inequality for $Q$ occurs follows from an indirect argument. Indeed, if $Q$ satisfies one of the constraints defining $\mathcal{A}_{2}$ with equality, a comparison argument based on cutting and 
pasting arguments and a key inequality stemming from the splitting properties of $\mathcal{S}\left(a^{-}, a^{+}\right)$and $\mathcal{S}\left(a^{+}, a^{-}\right)$- see (4.3) and Proposition 4.4 of Section 4 - leads to a contradiction.

Before ending this discussion, some motivation for mountain pass solutions for (HS) is in order. Let $Q$ be a minimizer of (2.6) or (2.15). Then for all $j \in \mathbb{Z}$, the functions $Q(\cdot+j)$ are also local minimizers of $J$. This suggests that $J$ satisfies the geometrical assumption of the Mountain Pass Theorem. However as for $I$, the $(P S)$ condition fails and there are problems with the differentiability of $J$. It will be seen in the next section how these obstacles can be overcome.

\section{A mountain pass theorem}

This section discusses a variant of the Mountain Pass Theorem that can be used to obtain a better version of Theorem 2.4 for (PDE) as well as mountain pass solutions for (HS). To formulate the result, let $E$ be a real Hilbert space and $I: E \rightarrow \mathbb{R}$. As usual, assume

$\left(I_{1}\right) I \in C^{1}(E, \mathbb{R})$.

Next an analogue of the geometrical assumption of the Mountain Pass Theorem is required. Let $e_{0} \neq e_{1} \in E$ and set

$$
\Gamma=\left\{\gamma \in C([0,1], E) \mid \gamma(0)=e_{0}, \gamma(1)=e_{1}\right\}
$$

The new geometrical assumption is:

$\left(I_{2}\right) b=\inf _{\gamma \in \Gamma} \max _{s \in[0,1]} I(\gamma(s))>\max \left\{I\left(e_{0}\right), I\left(e_{1}\right)\right\}$.

The replacement for the $(P S)$ condition is more complicated:

$\left(I_{3}\right)$ There are constants, $b^{*}>b, \nu>0, r^{*}>0$ and a sequence, $\left(\mathcal{A}_{j}\right)_{j \in \mathbb{Z}}$ of disjoint subsets of $E$ such that

(i) $\mathcal{A} \equiv\left\{u \in E \mid\left\|I^{\prime}(u)\right\| \leq \nu, I(u) \leq b^{*}\right\} \subset \cup_{j \in \mathbb{Z}} \mathcal{A}_{j}$,

(ii) $\left\|\mathcal{A}_{i}-\mathcal{A}_{j}\right\| \geq 3 r^{*}$ if $i \neq j$,

(iii) $(P S)$ holds in $\mathcal{A}_{j}$ for each $j \in \mathbb{Z}$, i.e. if $\left(u_{k}\right)$ is a sequence in $\mathcal{A}_{j}$ with $I\left(u_{k}\right)$ bounded and $I^{\prime}\left(u_{k}\right) \rightarrow 0$, then $u_{k}$ has a convergent subsequence.

See Figure 3. Then we have the following critical point theorem:

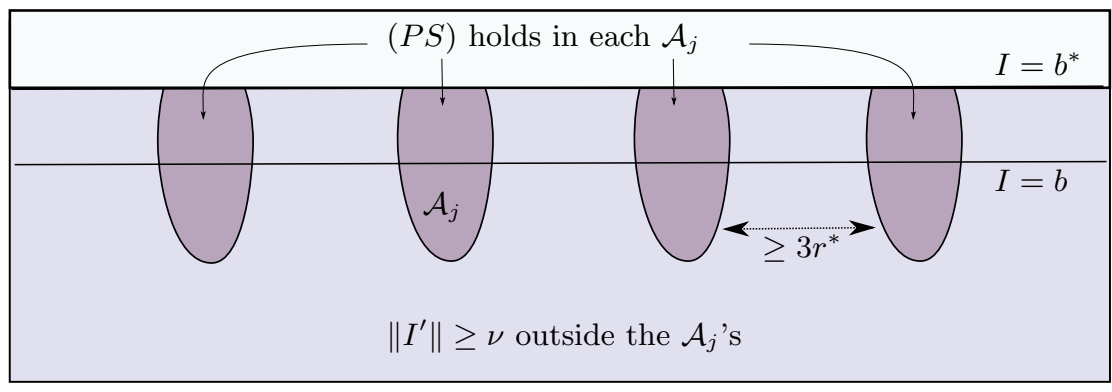

FiguRE 3. The assumption $\left(I_{3}\right)$ 
Theorem 3.1. Let $E$ be a real Hilbert space and $I: E \rightarrow \mathbb{R}$ with $I$ satisfying $\left(I_{1}\right)-\left(I_{3}\right)$. Then for any $\varepsilon>0, I$ possesses a critical value, $b_{\varepsilon} \in[b, b+\varepsilon)$ and a critical point, $u_{\varepsilon}$ with $I\left(u_{\varepsilon}\right)=b_{\varepsilon}$. Moreover $u_{\varepsilon}$ is not a local minimum of $I$.

The proof of Theorem 3.1 can be found in [7] (see Proposition 5.3). Note that unlike the Mountain Pass Theorem, it is not part of the conclusion that the minimax value, $b$, is a critical value of $I$. However if $b$ is not a critical value of $I$, it is the limit of a sequence of critical values so $I$ has infinitely many critical points. Next a simple example in which this phenomenon occurs will be presented. Let $g \in C^{2}(\mathbb{R}, \mathbb{R})$ satisfy the following conditions:

$\left(g_{1}\right)$ For $x \leq 0, g(x)=-1+\frac{1}{2}\left(\frac{\pi x}{2}\right)^{2}$.

$\left(g_{2}\right)$ For $x \in[0,3] \cup\{[4 j+1,4 j+3] \mid j \in \mathbb{N}\}, g(x)=\sin \frac{\pi}{2}(x-1)$.

$\left(g_{3}\right)$ For $x \in\left\{\left[4 j-1,4 j-\frac{1}{2}\right] \mid j \in \mathbb{N}\right\}, g$ is monotone decreasing.

$\left(g_{4}\right)$ For $x \in\left[4 j-\frac{1}{2}, 4 j+\frac{1}{2}\right]$ and $j \in \mathbb{N}, g(x)=-\alpha_{j}+(x-4 j)^{2}$, where $\left(\alpha_{j}\right)_{\mathrm{J} \in \mathbb{N}}$ is a sequence such that $\alpha_{j+1}>\alpha_{j}, \alpha_{1}>1$ and $\alpha_{j} \rightarrow 2$ as $j \rightarrow \infty$.

$\left(g_{5}\right)$ For $x \in\left\{\left[4 j+\frac{1}{2}, 4 j+1\right] \mid j \in \mathbb{N}\right\}, g$ is monotone increasing with $g(4 j+1)=0$. (See Figure 4 below).

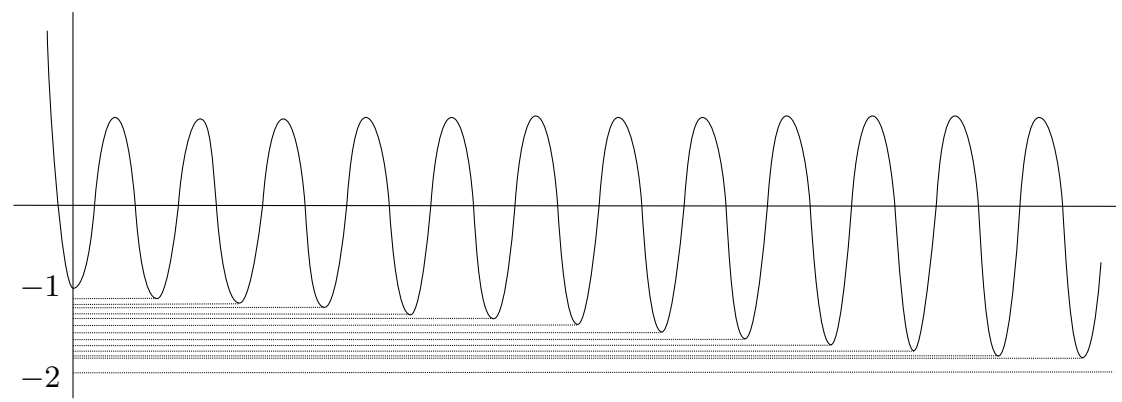

Figure 4. A possible function $g$

Now take $E=\mathbb{R}^{2}$ and define

$$
I(x, y)=2+g(x)-y^{2} .
$$

Clearly $I$ satisfies $\left(I_{1}\right)$. Take $e_{0}=(0,-2)$ and $e_{1}=(0,2)$. Then $I\left(e_{0}\right)=-3=I\left(e_{1}\right)$ while with $\Gamma$ and $b$ as defined above, it is easily seen that $b=0$ so $\left(I_{2}\right)$ holds. For $j \in \mathbb{N}$, setting $\mathcal{A}_{j}=\left[4(j-1)-\frac{1}{2}, 4(j-1)+\frac{1}{2}\right] \times\left[-\frac{1}{2}, \frac{1}{2}\right]$, it is straightforward to verify that $\left(I_{3}\right)$ holds. The critical points of $I$ are the points $\left.\{2(j-1), 0) \mid j \in \mathbb{N}\right\}$. When $j$ is even, they are local maxima with a common critical value of 3 ; when $j$ is odd, they are local mountain pass critical points of $I$ and the corresponding critical values are 1 when $j=1$ and $2-\alpha_{j}>0$ when $j>1$, all being positive. Therefore $b=0$ is not a critical value of $I$.

The proof of Theorem 3.1 is rather lengthy and technical and as mentioned above, can be found in [7]. To give a qualitative idea of the proof, let $\delta \in\left(0, r^{*}\right)$ and for $S \subset E$, let $\mathcal{N}_{\delta}(S)=\{u \in E \mid\|u-S\| \leq \delta\}$. The proof consists of four main 
steps. The first is to use $\left(I_{3}\right)$ and a related pseudogradient deformation to localize the search for critical points of $I$ to a small neighborhood, $\Omega=\mathcal{N}_{\delta}\left(\mathcal{A}_{j}\right)$, of one of the sets, $\mathcal{A}_{j}$, in which $(P S)$ holds. See Figure 5 below. Next a class of curves, $\Gamma_{\Omega}$ in

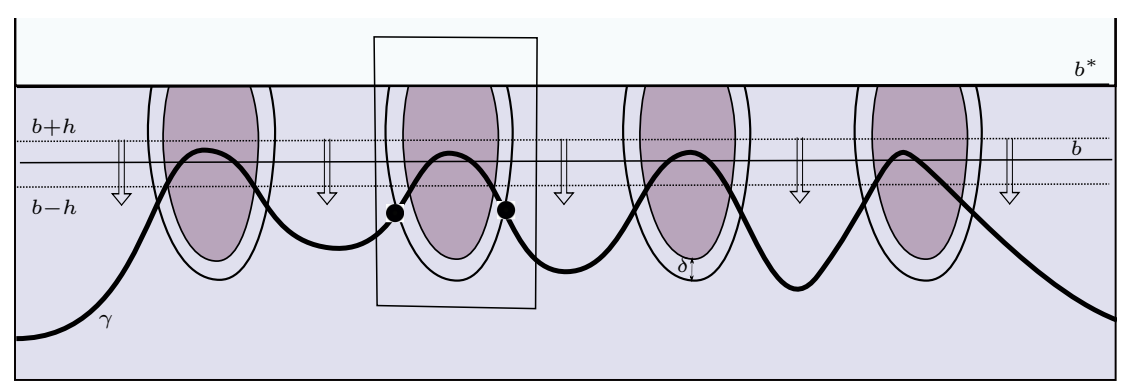

Figure 5. The assumption $\left(I_{3}\right)$ allows to use the gradient flow to select a new local MP structure

$\Omega$ is introduced and an associated minimax value, $b_{\Omega}$ for $I$ is defined. The choice of $\delta$ and the fact that these new curves are restrictions of some of the members of $\Gamma$ imply $b+h(\delta) \geq b_{\Omega} \geq b$ where $h(\delta) \rightarrow 0$ as $\delta \rightarrow 0$. Thirdly, by ( $\left.I_{3}\right)^{-}$-(iii), (PS) holds in $\mathcal{A}_{j}$. Thus a standard argument shows that $b_{\Omega}$ is a critical value of $I$ and there is a critical point, $U_{\Omega}$, of $I$ in $\Omega$ with $I\left(U_{\Omega}\right)=b_{\Omega}$. Lastly a local deformation argument shows $U_{\Omega}$ is not a local minimum of $I$.

Proposition 5.7 of [7] provides a more precise result than Theorem 3.1, namely:

Proposition 3.2. Under the same assumptions as Theorem 3.1, for any $\varepsilon \in\left(0, b^{*}-b\right)$, there exists a $b_{\varepsilon} \in[b, b+\varepsilon), a j_{\varepsilon} \in \mathbb{N}$, and a nonempty compact set, $\mathcal{K}_{\varepsilon} \subset \mathcal{A}_{j_{\varepsilon}}$, of critical points of $J$ having critical value $b_{\varepsilon}$. Moreover for $r^{*}$ as in $\left(I_{3}\right), \mathcal{K}_{\varepsilon}$ has the property that for each $r \in\left(0, r^{*} / 10\right)$, there is a $\lambda(r) \in\left(0,\left(b^{*}-b_{\epsilon}\right) / 4\right)$ such that whenever $h \in(0, \min \{\varepsilon, \lambda(r)\})$, there exists a pair of points $u_{0}(\delta, h), u_{1}(r, h)$ on $\partial B_{4 r}\left(\mathcal{K}_{\varepsilon}\right)$ and a path $\gamma_{r, h} \in C([0,1], E)$ joining $u_{0}(r, h)$ and $u_{1}(r, h)$ satisfying:

(i) $u_{0}(r, h), u_{1}(r, h) \in\left\{I \leq b_{\varepsilon}-h\right\}$;

(ii) $u_{0}(r, h), u_{1}(r, h)$ do not lie on a path in $N_{r^{*}}\left(\mathcal{K}_{\varepsilon}\right) \cap\left\{I<b_{\varepsilon}\right\}$;

(iii) $\gamma_{r, h}([0,1]) \subset \bar{N}_{4 r}\left(\mathcal{K}_{\varepsilon}\right) \cap\left\{u \in E \mid I(u)<b_{\varepsilon}+h\right\}$;

(iv) if $\operatorname{dist}_{E}\left(\gamma_{r, h}(\theta), \mathcal{K}_{\varepsilon}\right)>3 r$, then $\gamma_{r, h}(\theta) \in\left\{I(u) \leq b_{\varepsilon}-h\right\}$.

When $\mathcal{K}_{\varepsilon}$ consists of isolated points, Proposition 3.2 and the compactness of $\mathcal{K}_{\varepsilon}$ leads to the existence of a local mountain pass point in $\mathcal{K}_{\varepsilon}$ (see [2] - [6]). In more degenerate situations, Proposition 3.2 provides the existence of a local mountain pass structure inside of any small neighborhood of the entire set $\mathcal{K}_{\varepsilon}$ giving fruitful topological information about the local structure of the level sets of $I$ near $b_{\varepsilon}$.

In the following two sections, Theorem 3.1 will be applied to (HS) and (PDE). The case of (HS) will be treated first since it is somewhat simpler and more geometrical. 


\section{The application of Theorem 3.1 to (HS)}

Now we turn to the question of applying Theorem 3.1 to the setting of Example 2. This has been done in [7] where the complete details can be found. The arguments will merely be sketched here. For technical reasons, an additional hypothesis is needed for $V$ :

$\left(V_{4}\right) V \in C^{2}\left(\mathbb{R} \times \mathbb{R}^{m}, \mathbb{R}\right)$ and the matrix $V_{q q}\left(t, a^{ \pm}\right)=\left(\frac{\partial^{2} V}{\partial q_{i} \partial q_{j}}\left(t, a^{ \pm}\right)\right)$is positive definite uniformly in $t \in[0,1]$.

Next note that $J$ is not differentiable on $\mathcal{E}$ while $\left(I_{1}\right)$ of Theorem 3.1 requires differentiability. Thus a reformulation of $J$ is needed here. It was shown in [7] that $\{q \in \mathcal{E} \mid J(q)<\infty\}$ is a $C^{2}$ Hilbert manifold modeled on $E \equiv W^{1,2}\left(\mathbb{R}, \mathbb{R}^{m}\right)$ and consists of four components. Let $\psi_{1} \in \mathcal{M}\left(a^{-}, a^{+}\right)$and $\psi_{2} \in \mathcal{M}\left(a^{+}, a^{-}\right)$. Then the four components are given by $a^{ \pm}+E, \psi_{1}+E$, and $\psi_{2}+E$. Solutions of (HS) in $\psi_{1}+E$ correspond to heteroclinics from $a^{-}$to $a^{+}$, solutions in $\psi_{2}+E$ to heteroclinics from $a^{+}$to $a^{-}$, and solutions in $a^{ \pm}+E$ to homoclinics to $a^{ \pm}$. We will show there is a mountain pass heteroclinic solution in $\psi_{1}+E$. Related arguments yields mountain pass solutions for the other settings. More will be said about this point later.

Define a new functional, $I: E \rightarrow \mathbb{R}$ as follows. Let $q \in \Gamma\left(a^{-}, a^{+}\right)$. Then, with the aid of $\left(V_{4}\right),[7], u=q-\psi_{1} \in E$. Set

$$
I(u)=J(q)=J\left(u+\psi_{1}\right) .
$$

Then $I \in C^{2}(E, \mathbb{R})$ and satisfies $\left(I_{1}\right)$. We will indicate why $\left(I_{2}\right)-\left(I_{3}\right)$ are also satisfied. To verify $\left(I_{3}\right),\left(N D_{3}\right)$, a stronger nondegeneracy condition than $\left(N D_{2}\right)$ will be required and it will be introduced at that point. With the aid of $\left(N D_{3}\right)$, applying Theorem 3.1 yields

Theorem 4.2. Let $V$ satisfy $\left(V_{1}\right)-\left(V_{4}\right)$ and $\left(N D_{3}\right)$ hold. Then the functional, $I$ in (4.1) has a critical point in $E$ that is not a local minimum and (HS) possesses a corresponding mountain pass solution.

The verification of $\left(I_{2}\right)$ is based on two comparison arguments. First, since $\left(N D_{2}\right)$ holds, by (iii) of Proposition 2.10,

$$
4 r_{-}=\left\|K_{a^{-}}\left(a^{-}, a^{+}\right)-K_{a^{+}}\left(a^{-}, a^{+}\right)\right\|_{W^{1,2}\left(T_{0}, \mathbb{R}^{m}\right)}>0 .
$$

Define

$$
\Lambda\left(a^{-}, a^{+}\right)=\left\{q \in \Gamma\left(a^{-}, a^{+}\right) \mid\left\|q-K_{a^{-}}\left(a^{-}, a^{+}\right)\right\|_{W^{1,2}\left(T_{0}, \mathbb{R}^{m}\right)}=2 r\left(a^{-}, a^{+}\right)\right\},
$$

and

$$
d\left(a^{-}, a^{+}\right) \equiv \inf _{q \in \Lambda\left(a^{-}, a^{+}\right)} J(q)
$$

Then

Proposition 4.4. $d\left(a^{-}, a^{+}\right)>c\left(a^{-}, a^{+}\right)$.

Similarly, interchanging the roles of $a^{-}, a^{+}$gives a corresponding set, $\Lambda\left(a^{+}, a^{-}\right)$, and inequality

$$
d\left(a^{+}, a^{-}\right) \equiv \inf _{q \in \Lambda\left(a^{+}, a^{-}\right)} J(q)>c\left(a^{+}, a^{-}\right) .
$$


For the second comparison argument, recall that $\psi_{1} \in \mathcal{M}\left(a^{-}, a^{+}\right)$. Due to the nature of $\mathcal{M}\left(a^{-}, a^{+}\right)$and $\mathcal{S}\left(a^{-}, a^{+}\right)$, it can further be required that $\left.\psi_{1}(\cdot+i)\right|_{T_{0}} \in$ $K_{a^{-}}\left(a^{-}, a^{+}\right)$for $i \leq 0$ and $\left.\psi_{1}(\cdot+1)\right|_{T_{0}} \in K_{a^{+}}\left(a^{-}, a^{+}\right)$. Now a new comparison minimax value can be introduced by setting

$$
H\left(a^{-}, a^{+}\right) \equiv\left\{h \in C\left([0,1], \Gamma\left(a^{-}, a^{+}\right)\right) \mid h(0)=\psi_{1}(\cdot), h(1)=\psi_{1}(\cdot+1)\right\}
$$

and defining

$$
\hat{b}\left(a^{-}, a^{+}\right)=\inf _{h \in H\left(a^{-}, a^{+}\right)} \max _{s \in[0,1]} J(h(s))=\inf _{h \in H\left(a^{-}, a^{+}\right)} \max _{s \in[0,1]} I\left(h(s)-\psi_{1}\right) .
$$

Similarly reversing the roles of $a^{-}$and $a^{+}$gives another such minimax value, $\hat{b}\left(a^{+}, a^{-}\right)$. Then we have

\section{Proposition 4.7.}

$$
\hat{b}\left(a^{-}, a^{+}\right) \geq d\left(a^{-}, a^{+}\right) \text {and } \hat{b}\left(a^{+}, a^{-}\right) \geq d\left(a^{+}, a^{-}\right) .
$$

Thus by Proposition 4.4, (4.5) and (4.8), $\hat{b}\left(a^{-}, a^{+}\right)>c\left(a^{-}, a^{+}\right)$and $\hat{b}\left(a^{+}, a^{-}\right)>$ $c\left(a^{+}, a^{-}\right)$. Hence setting $e_{0}=0, e_{1}=\psi_{1}(\cdot+1)-\psi_{1}$ and $b=\hat{b}\left(a^{-}, a^{+}\right)$shows $I$ satisfies $\left(I_{2}\right)$ with $\Gamma=H\left(a^{-}, a^{+}\right)-\psi_{1} \subset E$. Likewise, reversing the roles of $a^{-}$and $a^{+}, I$ satisfies $\left(I_{2}\right)$ with the minimax level $b=\hat{b}\left(a^{+}, a^{-}\right)$.

It remains to verify $\left(I_{3}\right)$. This also requires several preliminaries. The first is a more elaborate analogue of Proposition 2.2 which analyzed the breakdown of $(P S)$ for (PDE). A closely related result obtains here. E.g. a $(P S)$ sequence in $\Gamma\left(a^{-}, a^{+}\right)$ will converge to a finite chain of solutions of (HS) starting at $a^{-}$and ending at $a^{+}$. To formulate this more precisely, let $\xi_{-}, \xi_{+} \in\left\{a^{-}, a^{+}\right\}$, let $\varphi$ be the appropriate member of $\left\{a^{ \pm}, \psi_{1}, \psi_{2}\right\}$, and $d>b$. Set

$$
\mathcal{D}^{d}\left(\xi_{-}, \xi_{+}\right)=\left\{\varphi+u \in \Gamma\left(\xi_{-}, \xi_{+}\right) \mid 0<I(u)=J(\varphi+u) \leq d \text { and } I^{\prime}(u)=0\right\} .
$$

Then we have:

Proposition 4.9. Suppose that $\left(q_{n}\right) \subset \Gamma\left(a^{-}, a^{+}\right)$where $q_{n}=\psi_{1}+u_{n}$ with $u_{n} \in E$. If further $I\left(u_{n}\right) \rightarrow \beta \geq c_{0}$ and $I^{\prime}\left(u_{n}\right) \rightarrow 0$ as $n \rightarrow \infty$, then there exists a $\kappa_{0}=$ $\kappa_{0}(\beta) \in \mathbb{N}$, an $l_{0} \in \mathbb{N} \cap\left[1, \kappa_{0}\right]$, points $\zeta_{1}, \zeta_{2}, \ldots ., \zeta_{l_{0}+1} \in\left\{a^{-}, a^{+}\right\}$with $\zeta_{1}=a^{-}$, $\zeta_{l_{0}+1}=a^{+}$, functions $U_{j} \in \mathcal{D}^{d}\left(\zeta_{j}, \zeta_{j+1}\right)$ for $j \in\left\{1, \ldots, l_{0}\right\}$, and sequences $\left(t_{n}^{j}\right) \subset \mathbb{Z}$, $j \in\left\{1, \ldots, l_{0}\right\}$, such that $t_{n}^{j+1}-t_{n}^{j} \rightarrow+\infty$ as $n \rightarrow+\infty$ having the property that along a subsequence of $n \rightarrow \infty$,

$$
\begin{aligned}
& \left\|q_{n}-U_{1}\left(\cdot-t_{n}^{1}\right)\right\|_{W^{1,2}\left(\left(-\infty, t_{n}^{1}+\frac{t_{n}^{2}-t_{n}^{1}}{2}\right), \mathbb{R}^{m}\right)} \rightarrow 0, \\
& \left\|q_{n}-U_{j}\left(\cdot-t_{n}^{j}\right)\right\|_{W^{1,2}\left(\left(t_{n}^{j}-\frac{t_{n}^{j}-t_{n}^{j-1}}{2}, t_{n}^{j}+\frac{t_{n}^{j+1}-t_{n}^{j}}{2}\right), \mathbb{R}^{m}\right)} \rightarrow 0 \quad j=2, \ldots, l_{0}-1, \\
& \left\|q_{n}-U_{l_{0}}\left(\cdot-t_{n}^{l_{0}}\right)\right\|_{W^{1,2}\left(\left(t_{n}^{l_{0}}-\frac{t_{n}^{l_{0}}-t_{n}^{l_{0}-1}}{2},+\infty\right), \mathbb{R}^{m}\right)} \rightarrow 0,
\end{aligned}
$$

and

$$
I\left(u_{n}\right)=J\left(q_{n}\right) \rightarrow \sum_{j=1}^{l_{0}} J\left(U_{j}\right) .
$$


Similarly if there is a (PS) sequence of $I$ on $\Gamma\left(\xi_{1}, \xi_{2}\right)$ for any pair $\xi_{1}, \xi_{2} \in$ $\left\{a^{-}, a^{+}\right\}$, the above result changes by replacing $a^{-}$by $\xi_{1}, a^{+}$by $\xi_{2}$, and $\psi_{1}$ by the appropriate member of $\left\{a^{ \pm}, \psi_{1}, \psi_{2}\right\}$.

Next an appropriate nondegeneracy condition is needed for solutions of (HS) having critical values below those of mountain pass solution(s). To formulate it, note first that $c\left(a^{-}, a^{+}\right)$and $c\left(a^{+}, a^{-}\right)$are positive. Set

$$
c\left(a^{ \pm}, a^{ \pm}\right)=\inf _{a^{ \pm}+u \in \mathcal{D}^{d}\left(a^{ \pm}, a^{ \pm}\right)} I(u) .
$$

As follows from Theorem 2.16, $\mathcal{D}^{d}\left(a^{-}, a^{-}\right) \neq \emptyset$ for large $d$ and interchanging the roles of $a^{-}$and $a^{+}$, the same is true of $\mathcal{D}^{d}\left(a^{+}, a^{+}\right)$. Then as was shown with the aid of $\left(V_{4}\right)$ in Proposition 3.5 of [7], $c\left(a^{ \pm}, a^{ \pm}\right)>0$. Thus (HS) cannot have a sequence of homoclinic solutions converging to $a^{-}$or $a^{+}$. Set

$$
c_{0}=\min _{\xi, \eta \in\left\{a^{-}, a^{+}\right\}} c(\xi, \eta)
$$

Now let $d>c_{0}$ and let $\mathfrak{D}^{d}$ denote the set of solutions, $Q$, of (HS) that are homoclinic or heteroclinic between $a^{-}$and $a^{+}$and such that $J(Q) \leq d$, i.e.

$$
\mathfrak{D}^{d} \equiv \mathcal{D}^{d}\left(a^{-}, a^{+}\right) \cup \mathcal{D}^{d}\left(a^{-}, a^{-}\right) \cup \mathcal{D}^{d}\left(a^{+}, a^{-}\right) \cup \mathcal{D}^{d}\left(a^{+}, a^{+}\right) .
$$

By our above remarks, $a^{-}, a^{+} \notin \mathfrak{D}^{d}$. Following what was done in Section 2, define

$$
\mathcal{S}^{d} \equiv\left\{\left.q\right|_{T_{0}} \mid q \in \mathfrak{D}^{d}\right\}
$$

Using the natural notation, as for $\mathfrak{D}^{d}, \mathcal{S}^{d}$ decomposes into four disjoint subsets:

$$
\mathcal{S}^{d}=\mathcal{S}^{d}\left(a^{-}, a^{+}\right) \cup \mathcal{S}^{d}\left(a^{-}, a^{-}\right) \cup \mathcal{S}^{d}\left(a^{+}, a^{-}\right) \cup \mathcal{S}^{d}\left(a^{+}, a^{+}\right)
$$

and $\overline{\mathcal{S}^{d}}$ possesses properties similar to those of $\overline{\mathcal{S}}\left(a^{-}, a^{+}\right)$, namely,

- $\overline{\mathcal{S}^{d}}=\mathcal{S}^{d} \cup\left\{a^{-}\right\} \cup\left\{a^{+}\right\}$,

- $\overline{\mathcal{S}^{d}}$ is compact in $W^{1,2}\left(T_{0}, \mathbb{R}^{m}\right)$.

Letting $\mathcal{C}^{d}\left(a^{ \pm}\right)$denote the component of $\overline{\mathcal{S}^{d}}$ to which $a^{ \pm}$belongs, Proposition 2.9 is replaced by

Proposition 4.10. One of the following mutually exclusive possibilities holds:

$$
\begin{aligned}
& 1^{o} \mathcal{C}^{d}\left(a^{ \pm}\right)=\left\{a^{ \pm}\right\} \\
& 2^{o} \mathcal{C}^{d}\left(a^{ \pm}\right)=\mathcal{C}^{d}\left(a^{\mp}\right), \\
& 3^{o} \mathcal{C}^{d}\left(a^{ \pm}\right) \neq\left\{a^{ \pm}\right\} \text {and } \mathcal{C}^{d}\left(a^{ \pm}\right) \subset \mathcal{S}^{d}\left(a^{ \pm}, a^{ \pm}\right) \cup\left\{a^{ \pm}\right\}
\end{aligned}
$$

The new nondegeneracy condition is that $1^{o}$ holds:

There is a $d>\hat{b}\left(a^{-}, a^{+}\right)$such that for $\xi \in\left\{a^{-}, a^{+}\right\}, \mathcal{C}^{d}(\xi)=\{\xi\}$.

As in Section 2, when $\left(N D_{3}\right)$ holds, $\mathcal{S}^{d}$ has an important separation property that plays a crucial role in the verification of $\left(J_{3}\right)$. For $j \in \mathbb{Z}$, set

$$
g_{j}: \overline{\mathcal{S}^{d}} \rightarrow \overline{\mathcal{S}^{d}}, \quad g_{j}\left(\left.q\right|_{T_{0}}\right)=\left.q(\cdot-j)\right|_{T_{0}} .
$$

Note that the function $g_{j}$ is continuous on $\overline{\mathcal{S}^{d}}$ and $g_{j}\left(a^{ \pm}\right)=a^{ \pm}$. 
Proposition 4.11. Suppose that $\left(\mathrm{ND}_{3}\right)$ holds. Then there exist closed nonempty disjoint subsets, $K_{1}^{-}, K_{2}^{-}$of $\overline{\mathcal{S}}^{d}$ such that $K_{1}^{-} \cup K_{2}^{-}=\overline{\mathcal{S}}^{d}$. Moreover $a^{-} \in K_{1}^{-}$and for any $z \in K_{1}^{-} \backslash\left\{a^{-}\right\}$, there exists a $p \in \mathbb{Z}$ such that $g_{p}(z) \in K_{2}^{-}$. Similarly there exist closed nonempty disjoint subsets, $K_{1}^{+}, K_{2}^{+}$of $\overline{\mathcal{S}}^{d}$ such that $K_{1}^{+} \cup K_{2}^{+}=\overline{\mathcal{S}}^{d}, a^{+} \in K_{1}^{+}$ and for any $z \in K_{1}^{+} \backslash\left\{a^{+}\right\}$, there exists a $p \in \mathbb{Z}$ such that $g_{p}(z) \in K_{2}^{+}$.

Now finally, we are ready to sketch the verification of $\left(I_{3}\right)$. Take $d=b^{*}>b$ and let $\nu$ and $r^{*}$ be small constants. Set

$$
\mathcal{A}=\left\{u \in E \mid\left\|J^{\prime}(u)\right\| \leq \nu, J(u) \leq b^{*}\right\} .
$$

We must construct the family of sets, $\mathcal{A}_{j}$ satisfying items (i)-(iii) of $\left(I_{3}\right)$. As a first step, determine $r_{0}$ from

$$
3 r_{0}=\min \left\{\operatorname{dist}_{W^{1,2}\left(T_{0}, \mathbb{R}^{m}\right)}\left(K_{1}^{-}, K_{2}^{-}\right), \operatorname{dist}_{W^{1,2}\left(T_{0}, \mathbb{R}^{m}\right)}\left(K_{1}^{+}, K_{2}^{+}\right)\right\}
$$

and define

$$
\begin{gathered}
\mathcal{K}_{0}\left(a^{-}\right)=\left\{\left.q\right|_{(-\infty, 2]}\left|q \in \mathcal{D}^{b^{*}}\left(a^{-}, a^{-}\right) \cup \mathcal{D}^{b^{*}}\left(a^{-}, a^{+}\right), q(\cdot+i)\right|_{T_{0}} \in K_{1}^{-}\right. \\
\text {for all } \left.i \leq 0, \text { and }\left.q(\cdot+1)\right|_{T_{0}} \in K_{2}^{-}\right\} .
\end{gathered}
$$

Thus $\mathcal{K}_{0}\left(a^{-}\right)$roughly consists of a piece of the unstable manifold of (HS) at $a^{-}$. As was shown in [7], $\mathcal{K}_{0}\left(a^{-}\right)$is compact in $W^{1,2}\left((-\infty, 2], \mathbb{R}^{m}\right)$. For $j \in \mathbb{Z}$, set

$$
\mathcal{K}_{j}\left(a^{-}\right)=\left\{\left.q(\cdot-j)\right|_{(-\infty, j+2]} \mid q \in \mathcal{K}_{0}\left(a^{-}\right)\right\},
$$

a phase shift of $\mathcal{K}_{0}\left(a^{-}\right)$. By (4.12), if $j_{1}<j_{2}, q_{1} \in \mathcal{K}_{j_{1}}\left(a^{-}\right)$, and $q_{2} \in \mathcal{K}_{j_{2}}\left(a^{-}\right)$, it follows that

$$
\left\|q_{1}-q_{2}\right\|_{W^{1,2}\left(\left[j_{1}+1, j_{1}+2\right], \mathbb{R}^{m}\right)} \geq 3 r_{0}
$$

Next for $j \in \mathbb{Z}$, define

$$
\mathcal{V}_{j}=\left\{u \in E \mid J(u) \leq b^{*} \text { and } \operatorname{dist}_{W^{1,2}\left((-\infty, j+2], \mathbb{R}^{m}\right)}\left(q, \mathcal{K}_{j}\left(a^{-}\right)\right)<r_{0}\right\}
$$

Hence $\mathcal{V}_{j}$ is a small neighborhood of part of the unstable manifold of (HS) at $a^{-}$. By (4.13), if $j_{1}<j_{2}$,

$$
\operatorname{dist}_{W^{1,2}\left(\mathbb{R}, \mathbb{R}^{m}\right)}\left(\mathcal{V}_{j_{1}}, \mathcal{V}_{j_{2}}\right) \geq r_{0}
$$

Due to Proposition 4.9, the family of sets, $\mathcal{V}_{j}$ also possess the important property that there is a constant, $\varepsilon_{0}>0$, such that

$$
\left\{u \in E \mid\left\|J^{\prime}(u)\right\| \leq \varepsilon_{0}, J(u) \leq b^{*}\right\} \subset \cup_{j \in \mathbb{Z}} \mathcal{V}_{j} .
$$

There is a similar family of sets with corresponding properties related to the stable manifold of (HS) at $a^{+}$that will be listed next. Set

$$
\begin{gathered}
\mathcal{K}_{0}\left(a^{+}\right)=\left\{\left.q\right|_{[0,+\infty)} \mid q \in \mathcal{D}^{b^{*}}\left(a^{+}, a^{+}\right) \cup \mathcal{D}^{b^{*}}\left(a^{-}, a^{+}\right)\right. \text {such that } \\
\left.\left.q(\cdot+j)\right|_{[0,1]} \in K_{2}^{-} \text {for any } j \geq 1 \text { and }\left.q\right|_{[0,1]} \in K_{1}^{-}\right\} .
\end{gathered}
$$

Then $\mathcal{K}_{0}\left(a^{+}\right)$is compact in $W^{1,2}\left([0,+\infty), \mathbb{R}^{m}\right)$. For each $j \in \mathbb{Z}$, let

$$
\mathcal{K}_{j}\left(a^{+}\right) \equiv\left\{\left.q(\cdot-j)\right|_{[j,+\infty)} \mid q \in \mathcal{K}_{0}\left(a^{+}\right)\right\} .
$$

If $j_{1}<j_{2} \in \mathbb{Z}, q_{1} \in \mathcal{K}_{j_{1}}\left(a^{+}\right)$, and $q_{2} \in \mathcal{K}_{j_{2}}\left(a^{+}\right)$, then

$$
\left\|q_{1}-q_{2}\right\|_{W^{1,2}\left(\left[j_{2}, j_{2}+1\right], \mathbb{R}^{m}\right)} \geq r_{0} .
$$


For $j \in \mathbb{Z}$, define

$$
\mathcal{W}_{j}=\left\{u \in E \mid J(u) \leq b^{*} \text { and } \operatorname{dist}_{W^{1,2}\left([j, \infty), \mathbb{R}^{m}\right)}\left(q, \mathcal{K}_{j}\left(a^{+}\right)\right)<r_{0}\right\} .
$$

Then by $(4.16)$, for $j_{1} \neq j_{2} \in \mathbb{Z}$,

$$
\operatorname{dist}_{W^{1,2}\left(\mathbb{R}, \mathbb{R}^{m}\right)}\left(\mathcal{W}_{j_{1}}, \mathcal{W}_{j_{2}}\right) \geq r_{0}
$$

As earlier, there is a constant, $\varepsilon_{1}>0$, such that

$$
\left\{u \in E \mid\left\|J^{\prime}(u)\right\| \leq \varepsilon_{1}, J(u) \leq b^{*}\right\} \subset \cup_{j \in \mathbb{Z}} \mathcal{W}_{j} .
$$

With the aid of these preliminaries, let $\varepsilon_{2}=\min \left\{\varepsilon_{0}, \varepsilon_{1}\right\}$ and define

$$
\hat{\mathcal{A}} \equiv\left\{u \in E \mid\left\|J^{\prime}(u)\right\| \leq \varepsilon_{2}, J(u) \leq b^{*}\right\} .
$$

The above remarks imply

$$
\hat{\mathcal{A}} \subset\left(\cup_{i \in \mathbb{Z}} \mathcal{V}_{i}\right) \cap\left(\cup_{j \in \mathbb{Z}} \mathcal{W}_{j}\right)
$$

so defining

(4.19) shows

$$
\mathcal{A}_{i, j}=\mathcal{V}_{i} \cap \mathcal{W}_{j} \quad i, j \in \mathbb{Z}
$$

$$
\hat{\mathcal{A}} \subset \cup_{i, j \in \mathbb{Z}} \mathcal{A}_{i, j}
$$

Moreover by (4.14) and (4.17), whenever $i, i^{\prime}, j, j^{\prime} \in \mathbb{Z}$ with $i \neq i^{\prime} \in \mathbb{Z}$ or $j \neq j^{\prime} \in \mathbb{Z}$, and each of $\mathcal{A}_{i, j}, \mathcal{A}_{i^{\prime}, j^{\prime}}$ are nonempty, then

$$
\left\|\mathcal{A}_{i, j}-\mathcal{A}_{i^{\prime}, j^{\prime}}\right\|_{E}=\operatorname{dist}_{W^{1,2}\left(\mathbb{R}, \mathbb{R}^{m}\right)}\left(\mathcal{A}_{i, j}, \mathcal{A}_{i^{\prime}, j^{\prime}}\right) \geq r_{0} .
$$

Consequently taking $\nu=\varepsilon_{2}, r^{*}=r_{0} / 3$ and $\mathcal{A}=\hat{\mathcal{A}}$, (4.19) - (4.21) show that $\mathcal{A}$ is contained in a countable family of disjoint sets satisfying $(i)-(i i)$ of $\left(I_{3}\right)$. Lastly the arguments that go into the proofs of (4.15) or (4.18) plus a bit more - see [7] show that (iii) of $\left(I_{3}\right)$ also holds and Theorem 3.1 implies the following more refined version of Theorem 4.2:

Theorem 4.22. Suppose $V$ satisfies $\left(V_{1}\right)-\left(V_{4}\right)$. With $b=\hat{b}$ as defined following (4.8), assume $\left(N D_{3}\right)$ holds for some $d>b^{*}>b$. Then for any $\varepsilon>0$, (HS) possesses at least one solution, $Q_{\varepsilon}=\psi_{1}+U_{\varepsilon} \in \psi_{1}+E$, with $J\left(U_{\varepsilon}\right) \in[b, b+\varepsilon)$. Moreover $U^{*}$ is not a local minimum of $J$.

Remark 4.23. Theorem 4.22 and the arguments that go into it can be extended in several ways:

(i) Replacing $\psi_{1}$ by $\psi_{2}$ in the above arguments leads to a family of heteroclinic mountain pass solutions in $\psi_{2}+E$.

(ii) As was mentioned at the end of Section 2, if $Q$ is a minimizer of (2.15), then for any $j \in \mathbb{Z}, Q(t+j)$ is also a local minimizer of $J$. This pair of functions can be used to play the roles of $e_{0}$ and $e_{1}$ in $\left(I_{2}\right)$ of Theorem 3.1. Thus corresponding to each of the 2-transition local minima of $J$ in the sets $\mathcal{A}_{2}(\mathbf{m}, l)$ of Section 2, using minor modifications of the arguments of this section, there are families of 2-transition mountain pass homoclinic solutions of (HS) in $a^{ \pm}+E$. 
(iii) Since the arguments of Section 2 carry over to prove there are $k$-transition local minima of $J$ in sets of admissible functions, $\mathcal{A}_{k}(\mathbf{m}, l)$, of $k$-transition type, the mountain pass arguments likewise extend to provide associated mountain pass solutions of $k$-transition type.

For more details, see [7].

\section{The application of Theorem 3.1 to (PDE)}

Now we return to (PDE). Other than the local minimum, $u \equiv 0$, there are no obvious critical points of the functional, $I$. Theorem 2.4 provides at least one mountain pass solution (up to integer phase shifts) whenever the nondegeneracy condition $\left(N D_{1}\right)$ is satisfied. In this section, a new nondegeneracy condition will be introduced. It leads to an improved version of Theorem 2.4 as well as to extensions of the multibump results of [14].

The new nondegeneracy condition, $(N D 4)$, was motivated by the one employed in [7] and [8] and other settings such as [36] and [28]. Again we begin with snapshots of the family of solutions. Let $P_{0}=[0,1]^{n}$ and for $j \in \mathbb{Z}^{n}, P_{j} \equiv j+P_{0}$. With $\mathcal{D}^{d}$ as defined for $I$ in Section 2, define

$$
\mathfrak{S}^{d} \equiv\left\{\left.U\right|_{P_{0}} \mid U \in \mathcal{D}^{d}\right\}
$$

This subset of $W^{1,2}\left(P_{0}, \mathbb{R}\right)$ consists of the family of restrictions to $P_{0}$ of critical points of $I$ in $\mathcal{D}$ such that $I(U) \leq d$. Then as was the case for its namesake for $(H S), \overline{\mathfrak{S}^{d}}=\mathfrak{S}^{d} \cup\{0\}$ is a compact metric space under the metric obtained from $\|\cdot\|_{W^{1,2}\left(P_{0}, \mathbb{R}\right)}$. If $\mathcal{C}^{d}(0)$ denotes the component in $\overline{\mathfrak{S}^{d}}$ containing 0 and $\mathcal{R}^{d}=\{u \in$ $\left.\overline{\mathfrak{S}^{d}} \mid\|u\|_{W^{1,2}\left(T_{0}, \mathbb{R}^{m}\right)} \geq 2 \bar{\rho}\right\}$, either

$$
\left(1^{o}\right) \mathcal{C}^{d}(0)=\{0\} \quad \text { or }\left(2^{o}\right) \mathcal{R}^{d} \cap \mathcal{C}^{d}(0) \neq \emptyset .
$$

This is the version of the "All or Nothing" alternative in the current setting. Here $\bar{\rho}>0$ is a small constant chosen so that if $u, v \in E$, then

$$
\int_{\mathbb{R}^{n}}\left|F_{u}(x, u) v\right| d x \leq \frac{1}{2}\|u\|\|v\| \text { whenever } \sup _{k \in \mathbb{Z}^{n}}\|u\|_{P_{k}} \leq 2 \bar{\rho} .
$$

The precise definition of $\bar{\rho}$ can be found in Section 2 of [16].

Finally the new nondegeneracy condition is that alternative $\left(1^{o}\right)$ holds:

$$
\text { there exists a } d>c \text { such that } \mathcal{C}^{d}(0)=\{0\} .
$$

This leads to an improvement of Theorem 2.4:

Theorem 5.3. Suppose that $(F 1)-(F 4)$ are satisfied. Let $c$ be defined by $(1.1), d>c$ and assume $\left(\mathrm{ND}_{4}\right)$ holds. Then for any $\varepsilon \in(0, d-c)$, (PDE) possesses a solution, $U_{\varepsilon}$, such that $I\left(U_{\varepsilon}\right) \in[c, c+\varepsilon)$ and $U_{\varepsilon}$ is not a local minimum of $I$.

The proof of Theorem 5.3 is via the use of Theorem 3.1 in the spirit of the proof of the proof of Theorem 4.22 and we refer the reader to [16] for details. 
Remark 5.4. To see that Theorem 5.3 is indeed a generalization of Theorem 2.4, suppose that $\left(N D_{1}\right)$ holds. Applying Theorem 5.3 for a sequence, $\varepsilon_{i} \rightarrow 0$ as $i \rightarrow \infty$ produces a sequence of critical points, $u_{i}$, of $I$ with $I\left(u_{i}\right) \rightarrow c$ as $i \rightarrow \infty$. Due to $\left(N D_{1}\right)$, this is only possible if $c$ is a critical value of $I$.

Next the existence of multibump solutions of (PDE) will be considered. This question was first studied in [14]. As already mentioned, if $u$ is a solution of (PDE), so is $u(\cdot+p)$ for any $p \in \mathbb{Z}^{n}$. Thus we seek solutions modulo this $\mathbb{Z}^{n}$ symmetry, i.e. solutions in $C^{2}\left(\mathbb{R}^{n}, \mathbb{R}\right) / \mathbb{Z}^{n}$. Theorem 1.9 of [14] showed:

Theorem 5.5. If $F$ satisfies $\left(F_{1}\right)-\left(F_{4}\right)$ and $\left(N D_{1}\right)$ holds, then for any $k \in \mathbb{N}$ and $\alpha>0$, there are infinitely many distinct solutions of $(\mathrm{PDE})$ in $C^{2}\left(\mathbb{R}^{n}, \mathbb{R}\right) / \mathbb{Z}^{n}$ having critical values in $(k c-\alpha, k c+\alpha)$.

The main existence result, Theorem 3.27, of [14] gives a sharper version of Theorem 5.5 under the same hypotheses. To avoid undo explanations, we will merely describe Theorem 3.27 more qualitatively. By Theorem 2.4, there are a finite number of solutions of $(\mathrm{PDE}), v_{1}, \ldots, v_{j}$ with $I\left(v_{p}\right)=c, 1 \leq p \leq j$. Due to the $\mathbb{Z}^{n}$ symmetry of $I$, these solutions can be normalized so that the maximum of $\left|v_{p}\right|$ occurs in $P_{0}$. Let $k \in \mathbb{N}$ and consider $\Sigma_{1}^{k} \varphi_{p}\left(x-l_{p}\right)$ where $\varphi_{p} \in\left\{v_{1}, \ldots, v_{j}\right\}, l_{1}, \ldots, l_{k} \in Z^{n}$, and $\left|l_{i}-l_{p}\right| \geq l_{0}=l_{0}(k)$ sufficiently large for $1 \leq i \neq p \leq k$. The main result in [14] showed there is an $r_{0}>0$ such that for any $r \in\left(0, r_{0}\right)$, there is a solution of (PDE) within $r$ of $\Sigma_{1}^{k} \varphi_{p}\left(x-l_{p}\right)$ (in the norm of $E$ ) for all but a finite number of choices of such sums. The proof of this result was indirect. Assuming the result was false, a deformation argument ultimately led back to a contradiction of Theorem 2.4

Now we turn to a generalization of these results of [14] given in [17] using the weaker nondegeneracy condition $\left(N D_{4}\right)$. Before stating a theorem, a few preparatory remarks are needed. As was mentioned above, Theorem 5.3 follows from Theorem 3.1 whose proof reduces the search for a critical point of $I$ to one of the sets, $\mathcal{A}_{q}$ as given via $\left(I_{3}\right)$. Keeping in mind that in $\left(N D_{4}\right), d>c$, choose $\varepsilon \in(0, d-c)$ in Theorem 3.1 obtaining a corresponding critical value, $\bar{c} \in[c, c+\varepsilon)$. More precisely, by Proposition 3.2, there is a set , $\mathcal{A}_{q}$, containing a compact set, $\mathcal{K}_{\bar{c}}$, of critical points of $I$ having $\bar{c}$ as critical value and satisfying (i)-(iv) of that Proposition. For a set $X \subset E$ and $p \in \mathbb{Z}^{n}$, let $f_{p}(X)=\{u(\cdot-p) \mid u \in X\}$ and let $\mathcal{B}_{\rho}(X)$ denote an open $\rho$-neighborhood of $X$ in $E$. For $y \in \mathbb{R}^{n}$, let $B_{\rho}(y)$ denote an open ball of radius $\rho$ about $y$ in $\mathbb{R}^{n}$. Lastly let $r^{*}$ be as in (ii) of $\left(I_{3}\right)$.

Now from [17], we have the following alternative:

Theorem 5.6. Suppose that $(F 1)-(F 4)$ are satisfied and $c$ is defined by (1.1). Let $d>c$ and assume $\left(N D_{4}\right)$ holds. Let $\bar{c}$ and $K \equiv \mathcal{K}_{\bar{c}}$ be as above. Then one of the following two alternatives occurs:

$1^{o}$ There exists an $a$ and $b$ in $\mathbb{R}$ such that $\bar{c} \in[a, b]$ and for any $s \in[a, b]$ there is a $U_{s} \in \mathcal{B}_{r^{*}}(K)$ such that $I\left(U_{s}\right)=s$ and $I^{\prime}\left(U_{s}\right)=0$;

$2^{o}$ For any $\delta>0$, there exists an $\overline{\mathcal{L}}=\overline{\mathcal{L}}(\delta)>0$ such that for any given $k \in \mathbb{N}$ with $k \geq 2$, and set of points, $\xi_{1}, \ldots, \xi_{k} \in \mathbb{Z}^{n}$ with $\left|\xi_{j}-\xi_{i}\right|>3 \overline{\mathcal{L}}$ when $i \neq j$, there is a solution $U \in E$ of ( $\mathrm{PDE})$ satisfying 
(i) $\left\|U-f_{\xi^{j}}(K)\right\|_{W^{1,2}\left(B_{\overline{\mathcal{L}}}\left(\xi_{j}\right)\right)}<\delta$ for each $j \in\{1, \ldots, k\}$, and

(ii) $\|U\|_{W^{1,2}\left(B_{\overline{\mathcal{L}}}(x) \backslash\left(\cup_{j=1}^{k} B_{\overline{\mathcal{L}}}\left(\xi_{j}\right)\right)\right)}<\delta$ for each $x \in \mathbb{R}^{n}$.

For either case $1^{o}$ or $2^{\circ}$, I has infinitely many distinct critical values.

By Theorem 5.6, either the set of critical values of $I$ near $\bar{c}$ is highly degenerate in the sense of $1^{o}$ or one can construct infinitely many multibump solutions of (PDE) as in $2^{o}$. Moreover due to $2^{o}(i)$, the solution $U$ is close to $f_{\xi_{j}}(K)$ on $B_{\overline{\mathcal{L}}}\left(\xi_{j}\right)$ for any $j \in\{1, \ldots, k\}$ and it is near 0 outside of $\cup_{j=1}^{k} B_{\overline{\mathcal{L}}}\left(\xi_{j}\right)$ in the sense of $2^{o}(i i)$.

When $1^{o}$ fails to occur, by fixing any sequence of points $\left(\xi_{i}\right)_{i \in \mathbb{N}}$ in $\mathbb{Z}^{n}$ such that $\left|\xi_{j}-\xi_{i}\right|>3 \overline{\mathcal{L}}$ when $i \neq j$, for any $k \in \mathbb{N}$, there is a solution $U_{k}$ of (PDE) satisfying $2^{o}(i)$ and $2^{o}(i i)$ with respect to the points $\left\{\xi_{1}, \ldots, \xi_{k}\right\}$. Then, a limit procedure gives the existence of solution to (PDE) having infinitely many bumps. See [17]

The proof of Theorem 5.6 involves an adaption to the present setting of some of the ideas originally developed in the earlier papers [18], [20], [19], [22] for ODE systems and in [14], [15] for (PDE) when $m=1$. Theorem 5.6 differs from the results in the earlier papers in two significant ways. Firstly, the set $\mathcal{K}_{\bar{c}}$ here is merely compact. As a result, a more complicated construction than in the cases previously studied is required. In addition, as was noted earlier, unlike the previous papers on (PDE) when $n>1$, our construction allows us to obtain the existence of " $k$ bump solutions", whenever the (appropriately measured) distance between the 1-bump solutions is sufficiently large, independently of the choice of $k \in \mathbb{N}$. Consequently by limit arguments, this result gives the existence of infinite-bump solutions to (PDE).

\section{A third example}

In this final section, (Per) will be discussed and some open questions will be posed. Recall

$$
-\Delta u+G_{u}(x, u)=0
$$

where $G$ satisfies

$\left(G_{1}\right) \quad G \in C^{2}\left(\mathbb{R}^{n} \times \mathbb{R}^{m}, \mathbb{R}\right)$ and is 1-periodic in all of its variables.

This system has many features in common with (HS), but as will be seen below, it has a richer and more complex structure of solutions. Most studied here is the case of $m=1$, a single equation. This equation is a special case of a more general quasilinear equation introduced by Moser [31] as a step towards developing a version for partial differential equations of the work of Aubry [42] and Mather [23] on self maps of an annular region in $\mathbb{R}^{m}$. When $n=1$ and $m \geq 1$, (Per) is an example of the dynamical systems to which the standard Aubry-Mather Theory applies. As in that theory, Moser sought solutions of (Per) that had special properties. They were minimal and without self intersection; see [31]. Moser's work was further developed by Bangert [32] and several others. Rabinowitz and Stredulinsky arrived at (Per) with $m=1$ from a different perspective. They were led to it in their research on 
Allen-Cahn models of spatial phase transitions. It turned out that these models could be viewed as special cases of (Per).

We will describe some of the results from their monograph, [33]. It begins by following Moser [31], to obtain an ordered family, $\mathcal{M}_{0}$, of solutions that are periodic in $x_{1}, \ldots, x_{n}$ and are minimizers of the functional associated with (Per) in that class of functions. Due to this ordering property, the union of the graphs of the members of $\mathcal{M}_{0}$ is either (i) all of $\mathbb{R}^{n+1}$, i.e. a foliation of $\mathbb{R}^{n+1}$ or (ii) there are functions, $v<w$ in $\mathcal{M}_{0}$ with no other members of $\mathcal{M}_{0}$ lying between them. As an example of (i), take $G \equiv 0$. For a simple example of (ii), set $n=m=1$ and consider the nonlinear pendulum model

$$
-\ddot{u}+a(x) \sin (2 \pi u)=0
$$

where the function $a(x)$ is positive and 1-periodic. Hence we can take $v=0$ and $w=1$. When (ii) occurs, we say $v$ and $w$ are a gap pair. Note that by $\left(G_{1}\right), v+j$ and $w+j$ are then also a gap pair for any $j \in \mathbb{Z}$.

The set of gap pairs of $\mathcal{M}_{0}$ play the role here of $a^{-}$and $a^{+}$for (HS) and 0 for (Per). They are the starting point for a complex hierarchy of solutions. Next in line to $\mathcal{M}_{0}$ are an enormous collection of solutions that are heteroclinic from $v$ to $w$ (and from $w$ to $v$ ) and lie between $v$ and $w$. In particular, there are solutions of (Per) in this region that are heteroclinic in $x_{1}$ from $v$ to $w$ and are 1-periodic in $x_{2}, \ldots, x_{n}$. The same is true for any permutation of the coordinates $x_{i}^{\prime}$. In fact, there are heteroclinics from $v$ to $w$ in any direction of the form $p=\left(p_{1}, \ldots, p_{n}\right) \neq 0$ where $p_{i} \in \mathbb{Z}, 1 \leq i \leq n$, the solutions being periodic in a related complementary set of directions. Each such family is obtained by the constrained minimization of a renormalized functional associated with (Per). See [33] for details.

As was the case for $\mathcal{M}_{0}$, once the direction of the heteroclinic is fixed, each of these families of solutions is ordered. For simplicity in what follows, we restrict ourselves to the family of heteroclinic solutions from $v$ to $w$ in the $x_{1}$ direction that are 1 -periodic in $x_{2}, \ldots, x_{n}$. We denote this family by $\mathcal{M}_{1}(v, w)$. There is a corresponding family of solutions, $\mathcal{M}_{1}(w, v)$, heteroclinic from $w$ to $v$. Like the analysis for $\mathcal{M}_{0}$, either the set of graphs of the members of $\mathcal{M}_{1}(v, w)$ is a highly degenerate set and foliates the region between $v$ and $w$ or merely laminates it in which case there is a gap pair $v_{1}<w_{1}$ in $\mathcal{M}_{1}(v, w)$. Assume that the latter case occurs and the same is true for its counterpart for $\mathcal{M}_{1}(w, v)$ with a gap pair of heteroclinics, $\bar{v}_{1}<\bar{w}_{1}$ in $\mathcal{M}_{1}(w, v)$. Then as was the case for (HS), there is a bifurcation of possibilities for further solutions. On the one hand, there are multitransition solutions that remain between the gap pair, $v, w$, and shadow members of $\mathcal{M}_{1}(v, w) \cup \mathcal{M}_{1}(w, v)$. On the other hand, there are also solutions that are heteroclinic in the $x_{1}$ direction from $v$ to $\bar{w}$ for some $\bar{w} \in \mathcal{M}_{0}$ with $\bar{w}>w$. For each case, these new solutions are 1-periodic in the other coordinate directions. The nonlinear pendulum given by (6.2) provides a physical example of these two possibilities. Translating the $\mathrm{x}$ variable, which represents time here, one can imagine a vertical pendulum with its weighted end lying above the origin that is released from rest at time $=-\infty$ and undergoes a to and fro motion relative to this vertical rest state terminating at time $=\infty$. This corresponds 
to the solutions of (6.2) that remain between the gap pair. Another possibility is a set of solutions that start as before but continue to rotate in the same direction until terminating again as earlier. These are the solutions that cross gaps.

The next stage of the hierarchy are solutions of (Per) that are heteroclinic in $x_{2}$ between the members of a gap pair, $v_{1}, w_{1}$ in $\mathcal{M}_{1}(v, w)$ and 1-periodic in the remaining coordinate directions.. They form an ordered set, $\mathcal{M}_{2}\left(v_{1}, w_{1}\right)$ and under an associated gap condition one can continue further in the hierarchy. See [32] or [33]. Each of the families of solutions mentioned above can be obtained by the constrained minimization of a renormalized functional associated with (Per) as was shown in [33]. A non variational approach to those families involving a single transition can be found in [32].

Remark 6.2. (i) It has been conjectured that there should exist a solution of (Per) corresponding to any geometrically feasible shape. This remains an open problem. (ii) When $m=1$, it is instructive to compare the use of gap pairs to that of the All or Nothing alternative as in Proposition 2.9. Indeed for the case of gap pairs, a stronger alternative occurs: either the graphs of the functions in $\mathcal{M}_{1}(v, w)$ form a foliation of $\left\{(x, y) \in \mathbb{R}^{n+1} \mid x \in \mathbb{R}^{n}, v(x) \leq y \leq w(x)\right\}$ or there is a gap pair of heteroclinic solutions between $v$ and $w$. In the first case $\mathcal{M}_{1}(v, w)$ is a continuum connecting $v$ and $w$ in $W^{1,2}\left([0,1] \times \mathbb{T}^{n-1}\right)$ while, the presence of a gap pair is equivalent to case (ii) of the All or Nothing Lemma.

Remaining in the setting of $m=1$, so far nothing has been said about mountain pass solutions of (Per). A wealth of such solutions also exist. The classical Mountain Pass Theorem shows there are infinitely many distinct periodic solutions between a gap pair in $\mathcal{M}_{0}$. Likewise if $v_{1}$ and $w_{1}$ is a gap pair in $\mathcal{M}_{1}(v, w)$, there is a mountain pass solution lying between them. This does not require Theorem 3.1 , but can be proved by another variant of the Mountain Pass Theorem based on the use of heat flow rather than negative gradient flow. See [34]. These methods also lead to some results on the variational gluing of mountain pass solutions and local minima. See [35] where they are called hybrid solutions. Many open questions concerning variational gluing remain when $m=1$.

Much less research has been done for (Per) when $m>1$. Part of the reason for this is that the Maximum Principle played an important role in proving many of the results mentioned above for the case of $m=1$ and it is no longer available when $m>1$. It is easy to show that there is an analogue of the periodic solutions, $\mathcal{M}_{0}$, here. Note that $u \in \mathcal{M}_{0}$ implies that $u+j \in \mathcal{M}_{0}$ for any $j \in \mathbb{Z}^{m}$. Under the further assumptions that

$$
G \text { is even in } x_{1}, \ldots, x_{n}
$$

and considering $\mathcal{M}_{0}$ modulo the the $Z^{m}$ symmetry of (Per), that

$$
\left(N D_{5}\right) \quad \mathcal{M}_{0} / \mathbb{Z}^{m} \text { is finite, }
$$

in [43] it was proved that for any $v, w \in \mathcal{M}_{0}$, there is a heteroclinic chain (in $x_{1}$ ) of solutions of (Per) joining $v$ and $w$, the solutions being periodic in $x_{2}, \ldots, x_{n}$. 
Moreover in [36], it was shown that if $\left(G_{1}\right)-\left(G_{2}\right)$ and $\left(N D_{5}\right)$ hold and for each of the links in the heteroclinic chain, an analogue of $\left(N D_{2}\right)$ is satisfied, then there are actual solutions of (Per) that shadow the heteroclinic chain.

In view of the enormous number of solutions that (Per) possesses when $m=1$ and of the similarities of this system with the system (HS), that despite the lack of a maximum principle possesses a mix of local minima and mountain pass solutions, we expect that there should be even more solutions for (Per) when $m>1$. Thus much further research remains to be done for this setting.

Funding. Open access funding provided by Università Politecnica delle Marche within the CRUI-CARE Agreement.

Open Access. This article is licensed under a Creative Commons Attribution 4.0 International License, which permits use, sharing, adaptation, distribution and reproduction in any medium or format, as long as you give appropriate credit to the original author(s) and the source, provide a link to the Creative Commons licence, and indicate if changes were made. The images or other third party material in this article are included in the article's Creative Commons licence, unless indicated otherwise in a credit line to the material. If material is not included in the article's Creative Commons licence and your intended use is not permitted by statutory regulation or exceeds the permitted use, you will need to obtain permission directly from the copyright holder. To view a copy of this licence, visit http://creativecommons.org/licenses/by/4.0/.

Publisher's Note. Springer Nature remains neutral with regard to jurisdictional claims in published maps and institutional affiliations.

\section{References}

[1] Ambrosetti, A, And Rabinowitz, P.H., Dual variational methods in critical point theory and applications, J. Functional Analysis 14 (1973), 349-381.

[2] Pucci, P. And Serrin, J., The structure of the critical set in the Mountain Pass Theorem, TransAMS 299 (1987), 115-132.

[3] Pucci, P. And Serrin, J., A Mountain Pass Theorem, J. Differential Equations 60 (1985), 142-149.

[4] Pucci, P. And Serrin, J., Extensions of the Mountain Pass Theorem, J. Funct. Anal. 59 (1984), 185-210.

[5] Hofer, H. A geometric description of the neighbourhood of a critical point given by the Mountain Pass Theorem, J. London Math. Soc. 31 (1985), 566-570.

[6] Caldiroli, P. And Montecchiari, P. Homoclinic orbits for second order Hamiltonian systems with potential changing sign, Commun. Appl. Nonlinear Anal. 1 (1994), 97-129.

[7] Montecchiari, P. And Rabinowitz, P.H. Solutions of mountain pass type for double well potential systems, Calc. Var. Partial Differential Equations 57 (2018), no. 5, paper no. $114,31 \mathrm{pp}$. 
[8] Montecchiari, P. And Rabinowitz, P.H. On global non-degeneracy conditions for chaotic behavior for a class of dynamical systems, Ann. IHP Anal. Nonlinéaire 36 (2019), no. 3, 627-653.

[9] Cieliebak, K. And SÉré, E., Pseudoholomorphic curves and the shadowing lemma, Duke Math. J. 99 (1999), 41-73.

[10] Coffman, C.V., A minimum-maximum principle for a class of nonlinear integral equations, J. Analyse Math. 22 (1969), 391-419.

[11] Coffman, C.V., On a class of nonlinear elliptic boundary value problems, J. Math. Mech. 19 (1970), 351-356.

[12] Hempel, J.A., Superlinear variational boundary value problems and nonuniqueness, thesis, Univ. of New England, Australia, 1970.

[13] Hempel, J. A., Multiple solutions for a class of nonlinear boundary value problems, Indiana Univ. Math J. 20 (1971), 983-996.

[14] Coti Zelati, V., and Rabinowitz, P. H., Homoclinic type solutions for a semilinear elliptic PDE on $\mathbb{R}^{n}$, Comm. Pure Appl. Math. 45 (1992), 1217-1269.

[15] Montecchiari, P., Multiplicity results for a class of Semilinear Elliptic Equations on $\mathbb{R}^{m}$, Rend. Sem. Mat. Univ. Padova 95 (1996), 1-36.

[16] Montecchiari, P. And Rabinowitz, P.H., A nondegeneracy condition for a semilinear elliptic system and the existence of 1- bump solutions, Discrete and Continuous Dynamical Systems - A 39, (2019), no. 12, 6995-7012.

[17] Montecchiari, P. And Rabinowitz, P.H., A nondegeneracy condition for a semilinear elliptic system and the existence of multibump solutions, submitted.

[18] SÉRÉ, E., Existence of infinitely many homoclinic orbits in Hamiltonian systems, Math. Z. 209 (1992), 27-42.

[19] SÉRÉ, E., Looking for the Bernoulli shift, Ann. IHP Anal. Nonlinéaire 10 (1993), 561590.

[20] Coti Zelati , V. And Rabinowitz, P.H., Homoclinic orbits for second order hamiltonian systems possessing superquadratic potentials, J. Amer. Math. Soc. 4 (1991), 693-727.

[21] S. Alama, S. And Li, Y., On "Multibump" Bound States for Certain Semilinear Elliptic Equations, Indiana J. Math. 41 (1992), 983-1026.

[22] Montecchiari, P., Existence and multiplicity of homoclinic solutions for a class of asymptotically periodic second order Hamiltonian systems, Ann. Mat. Pura ed App. CLXVIII (1995), 317-354.

[23] Mather, J.N., Existence of quasi-periodic orbits for twist homeomorphisms of the annulus, Topology 21 (1982), 457-467.

[24] Mather, J.N., Variational construction of connecting orbits, Ann. Inst. Fourier (Grenoble) 43 (1993), 1349-1386.

[25] Bolotin, S.V., Existence of homoclinic motions (Russian), Vestnik Moskov. Univ. Ser. 1 Mat. Mekh. (1983), 98-103.

[26] Kozlov, V.V., Calculus of variations in the large and classical mechanics, Russian Math. Surveys 40 (1985), 37-71.

[27] Sternberg, P., Vector-valued local minimizers of non-convex variational problems, Rocky Mt. J. Math. 21 (1991), 799-807. 
[28] Byeon, J., Montecchiari, P. And Rabinowitz, P.H., A double well potential system, APDE 9 (2016), 1737-1772.

[29] Alikakos, N.D. And Fusco, G., On the connection problem for potentials with several global minima, Indiana Univ. Math. J. 57 (2008), 1871-1906.

[30] Alessio, F., Montecchiari, P. And Zuniga, A., Prescribed energy connecting orbits for gradient systems, Discrete $\mathcal{E}$ Continuous Dynamical Systems - A 39 (2019), no. 8, 4895-4928.

[31] Moser, J., Minimal solutions of variational problems on a torus, Ann. IHP Anal. Nonlinéaire 3, (1986), 229-272.

[32] Bangert, V. , On minimal laminations of the torus, Ann. IHP Anal. Nonlinéaire 6 (1989), 95-138.

[33] Rabinowitz, P.H. And Stredulinsky, E., Extensions of Moser-Bangert Theory: Locally Minimal Solutions, Progress in Nonlinear Differential Equations and Their Applications, vol. 81, Birkhäuser, Boston, 2011.

[34] Bolotin, S. And Rabinowitz, P.H., A note on heteroclinic solutions of mountain pass type for a class of nonlinear elliptic PDE's, in: Progress in Nonlinear Differential Equations and Their Applications, vol. 66, Birkhäuser, Basel, 2006, pp. 105-114.

[35] Bolotin, S. And Rabinowitz, P.H., Hybrid mountain pass homoclinic solutions of a class of semilinear elliptic PDEs, Ann. IHP Anal. Nonlinéaire 31 (2014), 103-128.

[36] Montecchiari, P. And Rabinowitz, P.H., On the existence of multi-transition solutions for a class of elliptic systems, Ann. IHP Anal. Nonlinéaire 33 (2016), 199-219.

[37] Rabinowitz, P.H., A note on a semilinear elliptic equation on $\mathbb{R}^{n}$, in Nonlinear Analysis, A tribute in honour of G. Prodi, Quaderni Scuola Normale Superiore, Pisa (Ambrosetti, A. and Marino, A., eds.), 1991, pp. 307-317.

[38] Coti Zelati, V., Ekeland, I., And SÉRÉ, E., A Variational approach to homoclinic orbits in Hamiltonian systems, Math. Ann. 288 (1990), 133-160.

[39] Lions, P.-L., The concentration-compactness principle in the calculus of variations. The locally compact case, part 1, Ann. IHP Anal. Nonlinéaire 1 (1984), 109-145.

[40] Lions, P.-L., The concentration-compactness principle in the calculus of variations. The locally compact case, part 2, Ann. IHP Anal. Nonlinéaire 1 (1984), 223-283.

[41] Montecchiari, P. And Rabinowitz, P.H., A note on a class of double well potential problems, submitted.

[42] Aubry, S. and LeDaeron, P.Y., The discrete Frenkel-Kantorova model and its extensions I-Exact results for the ground states, Physica 8D (1983), 381-422.

[43] Rabinowitz, P.H., On a class of reversible elliptic systems, Networks and Heterogenious Media 7 (2012), 927-939.

Piero Montecchiari

Dipartimento di Ingegneria Civile, Edile e Architettura

Università Politecnica delle Marche,

Via brecce bianche, Ancona, I-60131, Italy

e-mail: p.montecchiari@univpm.it 
Paul H. Rabinowitz

Department of Mathematics

University of Wisconsin-Madison

Madison, Wisconsin, 53706, USA

e-mail: rabinowi@math.wisc.edu

Seminario Matematico e Fisico Lecture, delivered by Paul H. Rabinowitz on September 20, 2019

Received: June 22, 2020.

Accepted: July 26, 2020. 